\title{
Intestinal epithelial tuft cells initiate type 2 mucosal immunity to helminth parasites
}

François Gerbe ${ }^{1,2,3}$, Emmanuelle Sidot ${ }^{1,2,3}$, Danielle J. Smyth ${ }^{4}+$, Makoto Ohmoto ${ }^{5}$, Ichiro Matsumoto ${ }^{5}$, Valérie Dardalhon ${ }^{3,6}$, Pierre Cesses ${ }^{1,2,3}$, Laure Garnier ${ }^{1,2,3}$, Marie Pouzolles ${ }^{3,6}$, Bénédicte Brulin ${ }^{1,2,3}$, Marco Bruschi ${ }^{1,2,3}$, Yvonne Harcus ${ }^{4}$, Valérie S. Zimmermann ${ }^{3,6}$, Naomi Taylor ${ }^{3,6}$, Rick M. Maizels ${ }^{4} \dagger \&$ Philippe Jay ${ }^{1,2,3}$

Helminth parasitic infections are a major global health and social burden $^{1}$. The host defence against helminths such as Nippostrongylus brasiliensis is orchestrated by type 2 cell-mediated immunity ${ }^{2}$. Induction of type 2 cytokines, including interleukins (IL) IL-4 and IL-13, induce goblet cell hyperplasia with mucus production, ultimately resulting in worm expulsion ${ }^{3,4}$. However, the mechanisms underlying the initiation of type 2 responses remain incompletely understood. Here we show that tuft cells, a rare epithelial cell type in the steady-state intestinal epithelium ${ }^{5}$, are responsible for initiating type 2 responses to parasites by a cytokine-mediated cellular relay. Tuft cells have a Th2-related gene expression signature ${ }^{6}$ and we demonstrate that they undergo a rapid and extensive IL4R $\alpha$-dependent amplification following infection with helminth parasites, owing to direct differentiation of epithelial crypt progenitor cells. We find that the Pou $2 f 3$ gene is essential for tuft cell specification. Pou $2 \mathrm{f3}^{-/-}$mice lack intestinal tuft cells and have defective mucosal type 2 responses to helminth infection; goblet cell hyperplasia is abrogated and worm expulsion is compromised. Notably, IL-4R $\alpha$ signalling is sufficient to induce expansion of the tuft cell lineage, and ectopic stimulation of this signalling cascade obviates the need for tuft cells in the epithelial cell remodelling of the intestine. Moreover, tuft cells secrete IL-25, thereby regulating type 2 immune responses. Our data reveal a novel function of intestinal epithelial tuft cells and demonstrate a cellular relay required for initiating mucosal type 2 immunity to helminth infection.

Experimental subcutaneous infection of mice with $N$. brasiliensis $(\mathrm{Nb})$ stage 3 larvae induces a typical type- 2 response that involves a remodelling of epithelial cell populations, with goblet cell hyperplasia visible as soon as 5 days post-infection ${ }^{3,4}$. $N b$ L3 larvae first migrate from their injection site to the lungs, where they moult to the L4 stage, are coughed up, and swallowed to reach the intestines (day 2 post infection) where they mature and lay eggs (starting 5 days post-infection). $\mathrm{Nb}$ induces a rapid and robust type 2 response, resulting in worm expulsion by $6-8$ days post infection.

While the doublecortin-like kinase 1 (Dclk1)-expressing tuft cells represent only $0.4 \%$ of intestinal epithelial cells in naive mice ${ }^{5}$, we found that $\mathrm{Nb}$ infection resulted in a 8.5-fold expansion in tuft cells (Fig. 1a, b), first detected by 5 days post-infection in intestinal crypts, where proliferative epithelial progenitor cells reside, and also in the villi by 7 days post infection (Fig. 1c, Extended Data Fig. 1a). The kinetics of tuft cell expansion was equivalent to that of goblet cells (Fig. 1d, Extended Data Fig. 1b). Neo-differentiated tuft cells were indistinguishable from tuft cells present in naive mice, as evaluated by expression of established tuft cell markers, including Dclk1, Sry-related transcription factor 9 (Sox9), and phospholipase C gamma 2 (Plc $\gamma 2$ ) (Extended Data Fig. 1c $)^{6-8}$. All tuft cells, characterized by Dclk1 and growth factor independent $1 \mathrm{~b}(\mathrm{Gfil} b)^{8}$ expression also co-expressed the Pou domain, class 2, transcription factor 3 (Pou2f3) (Fig. 2a). In addition, rare $\left(<3 \%, n=400\right.$ cells counted) Pou $2 \mathrm{f}^{+}{ }^{+} ; \mathrm{Dclk} 1^{\text {low }}$ or Pou $2 \mathrm{f} 3^{+} ; \mathrm{Dclk} 1^{-}$cells
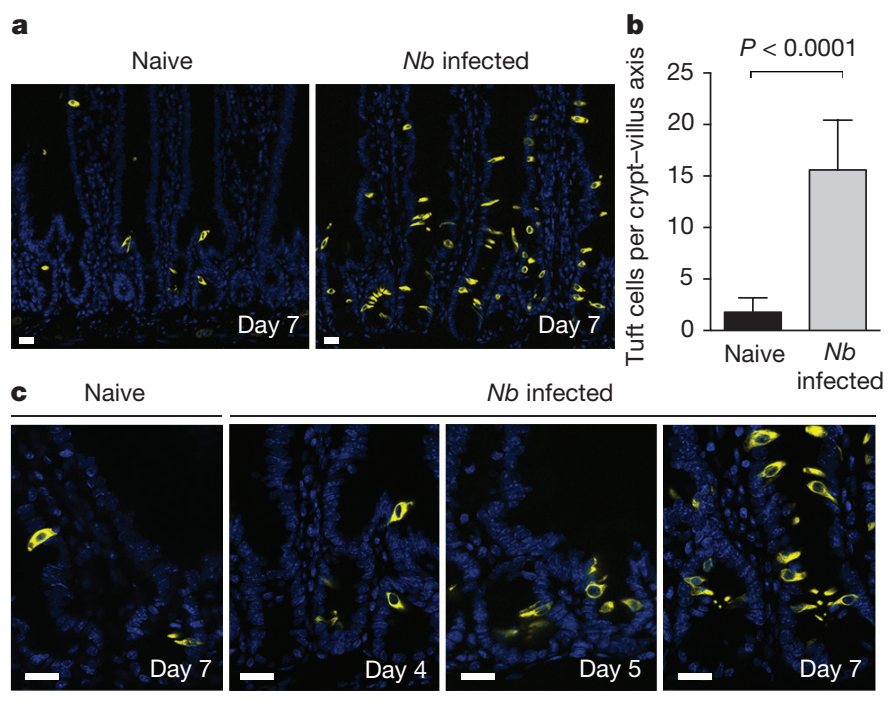

d Naive

Nb infected
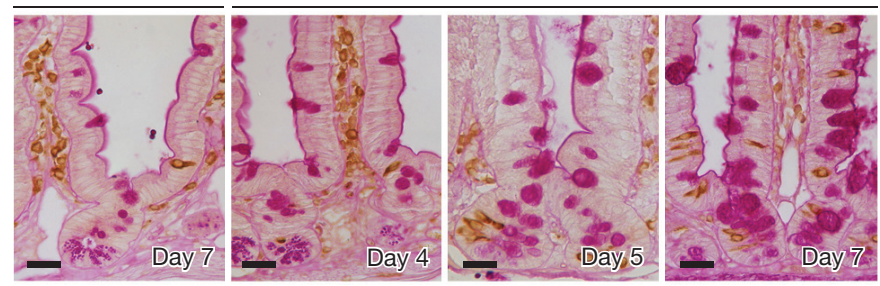

Figure 1 | Rapid amplification of the tuft cell lineage following infection with $N b$. a, Presence of tuft cells in the intestinal epithelia of naive and $\mathrm{Nb}$-infected mice 7 days post infection, visualized by expression of the Dclk1 marker. b, 8.7-fold increase of tuft cell numbers ( $1.8 \pm 1.4$ to $15.6 \pm 4.8$ per crypt-villus axis) in $N b$-infected mice compared to naive mice, 7 days post infection. ( $n=50$ crypt-villus units per mouse; 3 mice per condition). Data are shown as means \pm s.d. $(P<0.0001$, two-tailed Student's $t$-test with Welch's correction). c, Changes in the Dclk1-expressing tuft cell population in intestinal crypts are presented at the indicated time points post infection. Quantification is shown in Extended Data Fig. 1a. d, Corresponding goblet cell hyperplasia associated with numerous and larger mucus vacuoles, detected by periodic acid-Schiff (PAS) staining. Dclk1 cells are also visualized in brown. Quantification is shown in Extended Data Fig. 1b. Scale bars, $20 \mu \mathrm{m}$. All panels show representative pictures of experiments replicated 3 times in 3 mice per condition. 

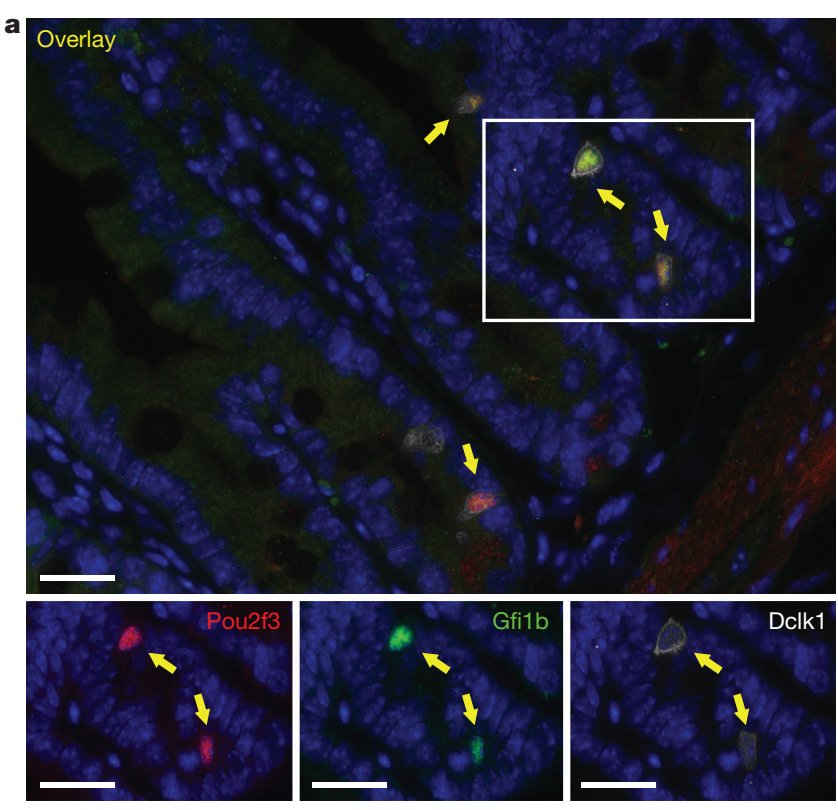

b
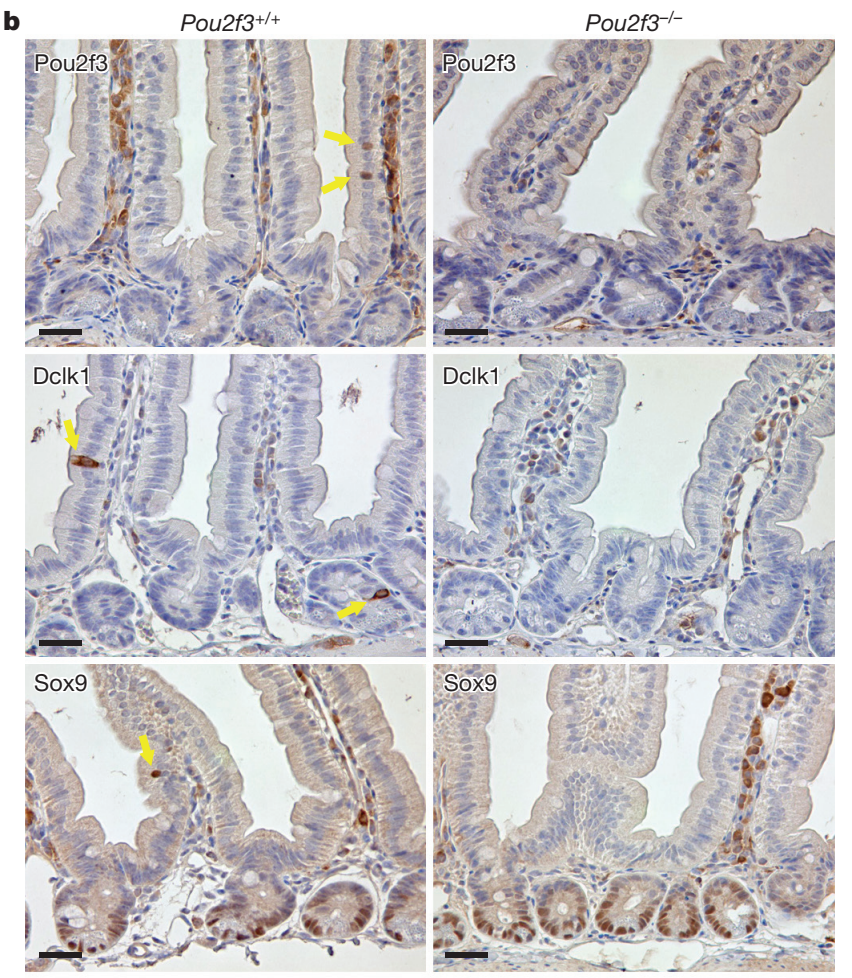

C

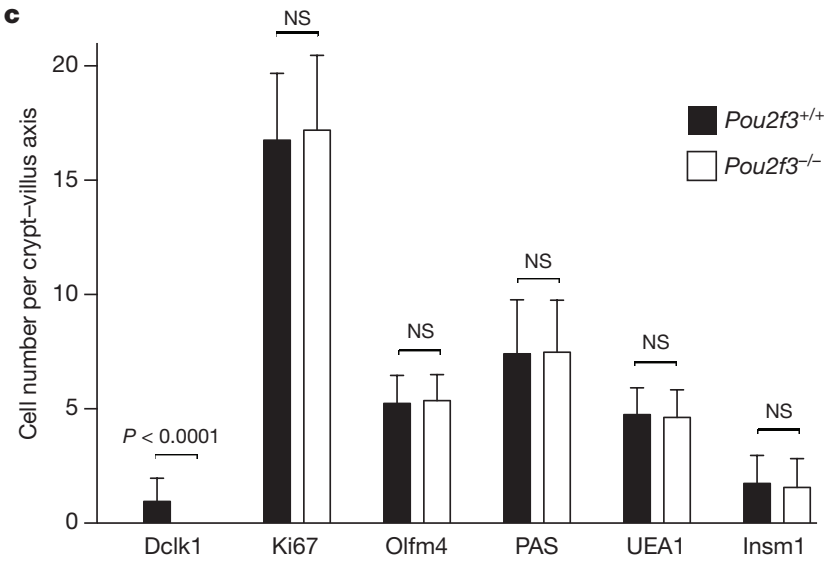

Figure $2 \mid$ Absence of tuft cells in the intestinal epithelium of Pou $2 \mathrm{f3}^{-/-}$ mice. a, Pou $2 \mathrm{f} 3$ is expressed specifically in tuft cells of the intestinal epithelium as determined by co-staining for Pou $2 \mathrm{f} 3$ and established markers of tuft cells such as Dclk1 and Gfilb. b, Pou $f 3$ deletion results in the absence of tuft cells as monitored by staining intestinal epithelium from Pou $2 \mathrm{f3}^{+/+}$and Pou $2 \mathrm{f3}^{-/-}$mice with Pou2f3-, Dclk1- and Sox9specific antibodies. a, b, Three mice per genotype were used for staining experiments. Scale bars, $20 \mu \mathrm{m}$. c, Pou $2 f 3$ deficiency does not affect the proliferation zone $(P=0.22)$, stem cell compartment $(P=0.66)$, enterocyte (not counted), goblet $(P=0.83)$, Paneth $(P=0.60)$ or enteroendocrine $(P=0.23)$ cell lineages as monitored by Ki67, Olfm 4 , alkaline phosphatase, PAS staining, UEA1 lectin, and Insm 1 , respectively. ( $n=50$ crypt-villus units per mouse; 3 Pou $23^{-/-}$and 3 wild-type mice). Data are shown as means \pm s.d. A two-tailed Student's $t$-test was used. Pictures show representative experiments replicated 3 times.

were found at the base of crypts, probably representing early differentiating tuft cells since villus Pou $2 \mathrm{f3}^{+}$cells always co-express Gfi1b and Dclk1. Following infection, the percentage of proliferating tuft cells in crypts increased from $13 \pm 5.6 \%$ to $24 \pm 14.9 \%(P=0.035)$, indicating that cell proliferation contributes to the amplification of the tuft lineage during type 2 responses (Extended Data Fig. 1d, e). Examination of the location of tuft cells present in $\mathrm{Nb}$ infected mice revealed that some tuft cells differentiate close to the stem cell zone (Extended Data Fig. 1d), suggesting that biased differentiation from the recently described Lgr $5^{+}$slowly cycling early secretory progenitors ${ }^{9}$ and $\mathrm{Dll}^{+}$secretory progenitors $^{10}$ also contributes to tuft cell lineage amplification. The increase in tuft cells was not due to a non-specific amplification of all secretory cell lineages as the number of enteroendocrine cells expressing the insulinoma-associated 1 ( $\operatorname{Insm} 1)$ marker ${ }^{11}$, another secretory lineage of the intestinal epithelium, was significantly $(P=0.008)$ reduced (Extended Data Fig. 1f, g).

To determine whether the increase in the tuft cell population following infection with $\mathrm{Nb}$ was specific to $\mathrm{C} 57 \mathrm{BL} / 6$ mice, we infected BALB/C mice and also observed a significant increase in tuft cell numbers (14-fold, $P<0.0001$; Extended Data Fig. 2a, b). Moreover, this response seems to be a common adaptation to helminth infection in general, as infection of C57BL/6 and BALB/c mice strains with Heligmosomoides polygyrus $^{12}$ also resulted in a significant increase in tuft cell numbers (6.1- and 8.3-fold, respectively, $P<0.0001$; Extended Data Fig. 2c, d). Tuft cell hyperplasia following $\mathrm{Nb}$ infection also occurred in $\mathrm{Rag}^{-/-}$ mice (10-fold; $P<0.0001)$ and therefore does not require functional adaptive immunity (Extended Data Fig. 2e, f).

Epithelial remodelling following helminth infection includes goblet cell hyperplasia and changes in mucus composition, associated with protective type 2 immunity ${ }^{13,14}$. To investigate the role of tuft cells in this process, we identified and characterized a tuft-cell-deficient mouse line. Mice deficient for the Pou2f3 transcription factor lack all Pou2f3-expressing taste receptor cells including sweet, umami and bitter taste cells ${ }^{15}$, as well as Trpm5-expressing chemosensory cells in the nasal cavity ${ }^{16}$ and olfactory epithelium ${ }^{17}$. Analysis of Pou $2 f 3$ deficient mice revealed a unique phenotype in the intestinal epithelium, with a complete absence of tuft cells as assessed by the absence of Pou2f3, Dclk1 and Sox9 expression outside the crypt compartment (Fig. 2b). The stem cell compartment, proliferation zone, and differentiation of enterocytes, goblet, enteroendocrine and Paneth cells were not affected (Fig. 2c and Extended Data Fig. 3). Furthermore, the distribution of immune cells in lymph nodes, mesenteric lymph nodes, spleen and lamina propria of Pou $2 \mathrm{f3}^{+/+}$and Pou $2 \mathrm{f3}^{-/-}$mice was equivalent (Extended Data Fig. 4) and lymphocytes were capable of responding to immune stimulation (Extended Data Fig. 5). Notably, type 2 innate lymphoid cells (ILC2s), a lineage that plays a critical role in secreting type 2 cytokines in response to helminth infection ${ }^{18,19}$, were present in both the mesenteric lymph nodes and lamina propria of Pou $23^{-1-}$ mice, at levels that were not significantly different from wild-type mice. (Extended Data Fig. 6a-c). Therefore, the absence of 

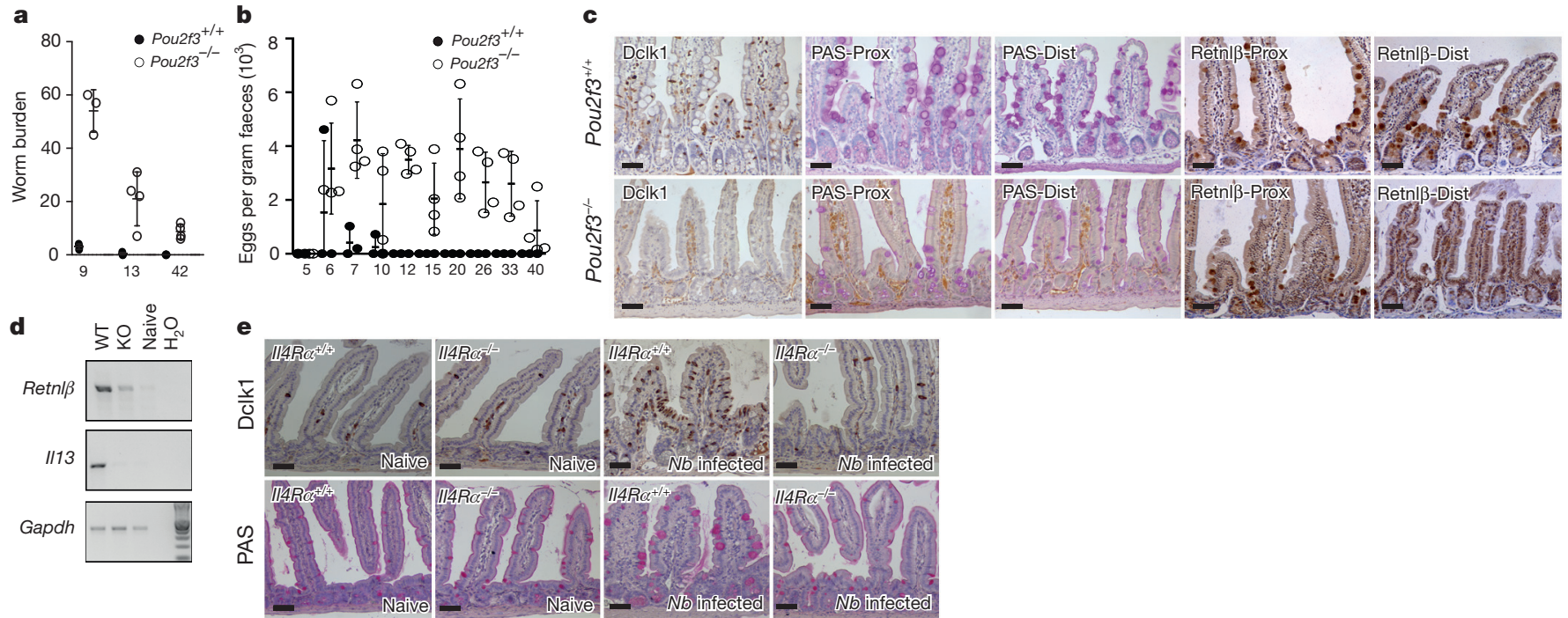

Figure 3 Impaired type 2 responses in tuft cell-deficient mice. a, Live adult worm counts in the small intestines of wild-type and Pou2 $\mathrm{f3}^{-1-}$ mice at days 9,13 and 42 post infection with $N b$ ( $n=3$ wild-type mice and 4 Pou $2 \mathrm{f3}^{-/-}$mice for each time point except for day 9 where $n=3$ Pou $2 \mathrm{f}^{-1-}$ mice). $\mathbf{b}$, Kinetic of $\mathrm{Nb}$ infection in 3 wild-type and 4 Pou $2 \mathrm{f3}^{-/}$mice, assessed by faecal eggs count. a, b, Each circle represents an individual mouse. The $x$ axis indicates time (days) post-infection. Average values \pm s.d. are shown. c, Immunohistochemistry illustrating the proximal and distal small intestinal epithelium of infected wild-type and Pou $2 \mathrm{f3}^{-1-}$ mice 7 days after infection ( $n=3$ mice per genotype).

Pou2f3 does not affect global immunity or intestinal epithelium formation. Rather, Pou2f3 represents the first identified transcription factor that is specifically required to specify the tuft cell lineage in the intestinal epithelium, analogously to Sox9 for Paneth ${ }^{20,21}$ and Ngn3 for enteroendocrine ${ }^{22}$ cell lineages. Thus, Pou $2 \mathrm{f3}^{-1-}$ mice represent a powerful model to study the function of tuft cells.

Pou $2 \mathrm{f3}^{+/+}$and Pou $2 \mathrm{f3}^{-/-}$mice were infected with $\mathrm{Nb}$ and analysed at several time points. In Pou $2 \mathrm{f}^{+/+}$mice, only few worms were found after 9 days and expulsion was nearly complete after 13 days. In sharp contrast, numerous worms were found in Pou $2 \mathrm{f3}^{-/-}$mice up to 42 days post infection (Fig. 3a, b), not only in the proximal part of the small intestine, their normal site of attachment ${ }^{23}$, but also in more distal locations. Together, these data strongly suggest that a compromised type- 2 response is responsible for prolonged worm survival in Pou $23^{-I-}$ tuft-cell-deficient mice.

To understand the mechanisms underlying the delayed worm expulsion in Pou2f3-deficient mice, we analysed the type- 2 responsedependent remodelling of the intestinal epithelium 7 days after infection, a time point at which adult worms were detected in all infected animals. In Pou $23^{+/+}$mice, the intestinal epithelium displayed extensive and generalized goblet cell hyperplasia, with large mucus vacuoles, and tuft cell hyperplasia (Fig. 3c). Expectedly, Pou $23^{-/-}$mice completely lacked tuft cells and, in contrast to Pou $2 \mathrm{f3}^{+/+}$mice, were devoid of overt goblet cell hyperplasia, with focal and moderate hyperplasia limited to the most proximal small intestine, and lower goblet cell numbers than wild-type mice (Fig. 3c, Extended Data Fig. 7a, and Supplementary Information 1 and 2). Therefore, tuft-cell-deficient mice have a delayed type 2 response, with deficient mucosal goblet cell hyperplasia and delayed control of $\mathrm{Nb}$ infection.

The goblet cell-produced Resistin-like beta (Retnl $\beta$ ) molecule, strongly induced by type 2 cytokines, has direct anti-helminth activity that facilitates expulsion ${ }^{3,24}$. We compared expression of Retnl $\beta$ in wild-type and Pou $2 \mathrm{f3}^{-/-}$mice 7 days after $\mathrm{Nb}$ infection, when worm expulsion had started in wild-type mice. Retnl $\beta$ was strongly expressed in hyperplastic goblet cells in Pou $23^{+/+}$mice, but was only weakly expressed in Pou $23^{-1-}$ mice (Fig. 3c, d and Extended Data Fig. 7a).
Dclk1 and PAS stainings, respectively, reveal tuft and goblet cells, as well as Retnl $\beta$ production. d, Quantification of IL- 13 and Retnl $\beta$ in the intestinal mucosa of naive, and $\mathrm{Nb}$-infected Pou $2 \mathrm{f3}^{+/+}$and Pou $2 \mathrm{f3}^{-/-}$mice by RT-PCR, 7 days after infection. Representative gels are shown with relative Gapdh expression presented as an internal control. e, Histological analysis showing tuft (Dclk1 staining) and goblet (PAS staining) cells in naive and

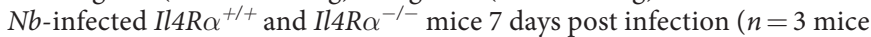
per genotype). Scale bars, $20 \mu \mathrm{m}$. All panels show representative pictures of experiments replicated 3 times.

Moreover, while IL-4 levels were equivalent in mucosal tissue of $\mathrm{Nb}$-infected Pou $2 \mathrm{f3}^{+/+}$and Pou $23^{-/-}$mice, IL-13 levels were markedly decreased in the latter (Fig. 3d). As both IL- 4 and IL-13 type 2 cytokines are known to regulate Retnl $\beta$ expression ${ }^{3}$, and IL- 4 is dispensable during type 2 responses to $N b^{25}$, our data strongly suggest that defective IL-13 production is responsible for the decreased Retnl $\beta$ expression in $\mathrm{Nb}$-infected Pou $23^{-/-}$mice. Thus, we identify a defective IL-13/ Retnl $\beta$ axis in tuft-cell-deficient mice with impaired worm expulsion.

We next studied the link between tuft cells and type-2-mediated mucosal adaptation following $N b$ infection. IL-4R $\alpha$ signalling is essential for both goblet cell hyperplasia and type 2 immune responses occurring upon helminth infection, and deletion of the Il4r $\alpha$ gene abrogates $N b$ expulsion ${ }^{23,26}$. Importantly, the $N b$-induced tuft cell hyperplasia occurring in wild-type mice 7 days post infection was absent in Il4r $\mathrm{r}^{-1-}$ mice, as was goblet cell hyperplasia (Fig. 3e, Extended Data Fig. 7b). This demonstrates the critical role of IL- $4 \mathrm{R} \alpha$ signalling in the expansion of the tuft cell population following helminth infection.

We then examined whether IL-4R $\alpha$ signalling is sufficient to trigger tuft cell lineage hyperplasia by injecting naive C57BL/6 mice with recombinant murine IL-4 and/or IL-13 (rIL-4/rIL-13) for 5 days and assessing the histology of the intestinal epithelium. rIL-4/rIL-13 injection induced goblet cell hyperplasia together with tuft cell expansion (Extended Data Fig. 7c). Importantly, treatment of Pou $2 \mathrm{f3}^{-/-}$mice with rIL-4/rIL-13 also resulted in goblet as well as Paneth cell hyperplasia, indicating a function of tuft cells upstream of IL-4/IL-13 (Extended Data Fig. 7c, d). Moreover, ectopic IL-4/IL-13 induced Retnl $\beta$ expression in goblet cells, independently of the Pou $2 f 3$ genotype. Retnl $\beta$ expression was found predominantly in crypts and was therefore delayed compared to the onset of goblet cell hyperplasia (Extended Data Fig. 7c), and quantitatively lower than in an infectious context (Fig. 3c). Thus, IL-4R $\alpha$ signalling is sufficient to induce an expansion of the tuft cell lineage. Furthermore, ectopic stimulation of this signalling cascade obviates the need for tuft cells in the epithelial cell remodelling of the intestine, including induction of Retnl $\beta$ expression by hyperplastic goblet cells.

To determine whether the IL-4/IL-13-induced goblet cell hyperplasia was epithelial-cell-autonomous, we used an ex vivo organoid culture 
a

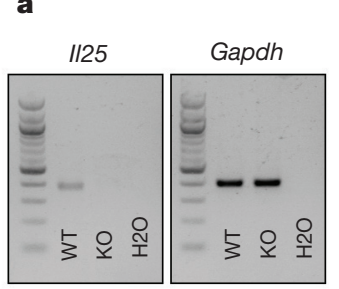

\section{b}

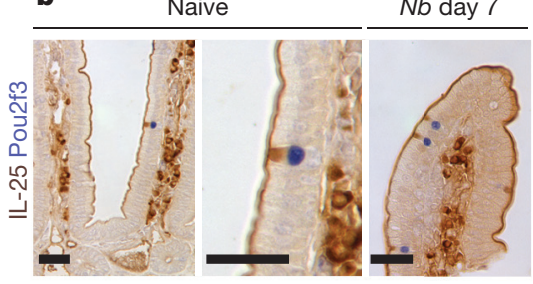

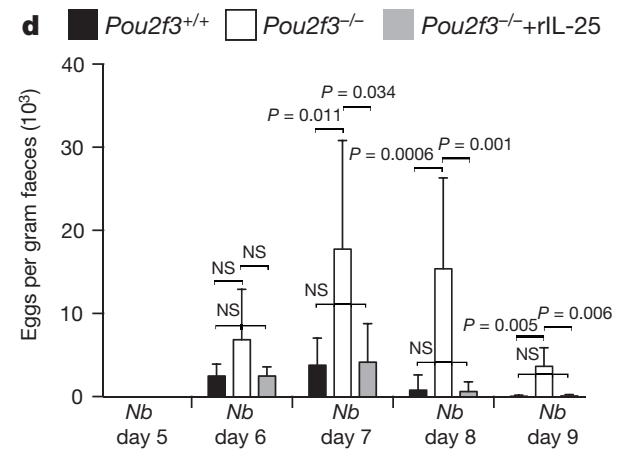

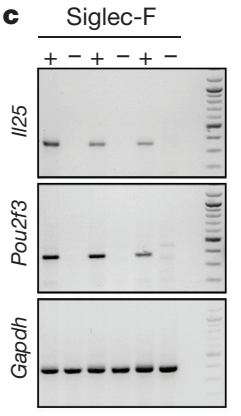

e

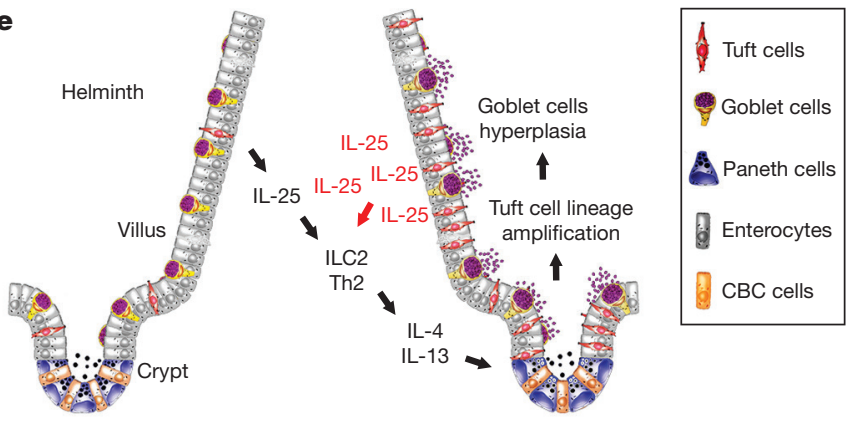

Figure 4 | Tuft cells express IL-25, and rIL-25 is sufficient to initiate type 2 mucosal responses in the absence of tuft cells. a, Analysis of Il25 mRNA expression in Pou $2 \mathrm{f3}^{+/+}$and Pou $2 \mathrm{f3}^{-/-}$mice infected with $\mathrm{Nb}$, 9 days post-infection, by RT-PCR. Gapdh expression is presented as an internal control. b, Immunohistochemistry showing IL-25 expression in naive and $\mathrm{Nb}$-infected wild type mice. Blue staining, nuclear Pou2f3 expression revealed with NBT/BCIP. Brown staining, IL-25 expression revealed with $\operatorname{DAB}(n=3$ naive and 3 infected mice). Scale bars, $20 \mu \mathrm{m}$. c, PCR with reverse transcription (RT-PCR) showing predominant Il25 and Pou $2 f 3$ mRNA expression in the FACS-enriched tuft cells fractions $(+)$ and the other epithelial cells $(-)$, obtained from 3 independent mice. Gapdh is shown as an internal control. d, Rescue of the Pou2f3 deficiency by treatment with exogenous rIL-25, as assessed by egg counts during a time course of infection with $\mathrm{Nb}$ ( $n=7$ mice for the wild-type and Pou $23^{-1-}$ mice, and $n=6$ for the rIL-25-treated Pou $2 \mathrm{f3}^{-/-}$mice). Average \pm s.d. are presented, as well as exact $P$ values when $<0.05$ (two-tailed Mann-Whitney $U$-test). e, Scheme illustrating the function of tuft cells in initiating type 2 responses following infection with intestinal helminths. Left, normal epithelium undergoing infection with a helminth. Right, tuft cell-dependent epithelial remodelling during type 2 responses. All panels show representative pictures of experiments replicated 3 times.

system $^{27}$ that allows physiological responses of an isolated intestinal epithelium to be analysed in the absence of stromal cues. As expected, tuft cells were absent in Pou $2 \mathrm{f3}^{-/-}$organoid cultures (Extended Data Fig. 8a). Moreover, in wild-type organoids, the tuft cell population increased as early as $48 \mathrm{~h}$ following addition of rIL-4/rIL-13 (Extended Data Fig. $8 \mathrm{a}, \mathrm{b}$ ). Treatment with rIL- 4 or rIL-13 alone yielded identical results to the rIL-4/rIL-13 mixture (Extended Data Fig. $8 \mathrm{c}$ ). Treatment of Pou $23^{-/-}$organoids with rIL-4/rIL-13 also triggered goblet cell hyperplasia equivalent to that detected in Pou $23^{+1+}$ organoids, as indicated by Retnl $\beta$ expression (Extended Data Fig. 8d), revealing the critical role of type 2 cytokine signalling downstream of the tuft cell lineage. Furthermore, these data demonstrate that the intestinal epithelial response to IL-4/IL-13 is epithelium-autonomous and does not require additional stromal signals. Together, our data identify a novel function of tuft cells in initiating the mucosal type 2 responses with a positive feedback loop through IL-13-producing immune cells that, in turn, amplify the tuft cell lineage.

Finally, we investigated the physiological function of the tuft cell hyperplasia, fully established by 7 days post-infection when worm expulsion starts. IL-25 is an alarmin molecule produced by an as yet unidentified intestinal epithelial cell type, capable of initiating type 2 responses by stimulating ILC2s to produce IL- 4 and IL-13 ${ }^{18,19}$. We thus analysed Il 25 messenger RNA expression in Pou $2 \mathrm{f3}^{+/+}$and Pou $2 \mathrm{f3}^{-/-}$ mice infected with $\mathrm{Nb}$. Nine days after infection, Il25 expression was higher in the intestinal mucosa of $\mathrm{Pou}_{2 \mathrm{f} 3^{+/+}}$mice than in tuft-celldeficient Pou $2 \mathrm{f3}^{-1-}$ mice (Fig. 4a). Moreover, IL-25 protein expression was restricted to tuft cells in naive mice (Fig. $4 \mathrm{~b}$ and Extended Data Fig. 9a) and consistent with these data, Il25 mRNA was only detected in the FACS-enriched tuft cell fraction of the intestinal epithelium (Fig. 4c and Extended Data Fig. 9b). Following $\mathrm{Nb}$ infection, IL-25 expression remained restricted to tuft cells (Fig. 4b). Concomitant with tuft cell hyperplasia, epithelial IL-25 expression peaks 9 days after infection with $N b$, at the time of worm expulsion, for which it is required ${ }^{28}$. In accord with a critical role for IL-25-secreting tuft cells in the expansion of ILC2s, we found that the percentage of $\mathrm{Lin}^{-} \mathrm{CD} 127^{+} \mathrm{Gata} 3^{+} \mathrm{KLRG}^{+}$ILC2s was not significantly augmented by $\mathrm{Nb}$ infection of Pou $2 \mathrm{f3}^{-/-}$mice, but was significantly augmented in wild-type mice. Indeed, tuft cells were required for the global induction of an adaptive immune response as helminth infection induced an approximately 2.5 -fold expansion of both ILC2 and Th2 subsets in mesenteric lymph nodes, whereas these subsets remained unchanged in the infected Pou $23^{-1-}$ mice $(P=0.02$, $P=0.0005$, respectively; Extended Data Fig. 6d-f). It is likely that these immune defects are directly due to the paucity of IL-25 as treatment of $\mathrm{Nb}$-infected Pou $2 \mathrm{f3}^{-/-}$mice with rIL-25 almost completely compensated for the absence of tuft cells, promoting an efficient worm expulsion (Fig. 4d). IL-25 thus provides a mechanistic link between tuft cells, promotion of type 2 responses and worm expulsion.

Taken together, our data reveal a critical function of tuft cells in initiating mucosal type 2 responses following infection with helminths through IL-25 secretion. In the absence of tuft cells, IL-25 and IL-13 expression remain low, and type 2 mucosal responses and worm expulsion are delayed. Our study demonstrates a requirement for tuft cells upstream of IL-4/IL-13, with these cytokines driving tuft cell hyperplasia, thereby amplifying a feed-forward loop to orchestrate a rapid and effective anti-helminth immunity (Fig. 4e).

Online Content Methods, along with any additional Extended Data display items and Source Data, are available in the online version of the paper; references unique to these sections appear only in the online paper.

\section{Received 19 August; accepted 10 December 2015.}

1. Hotez, P. J. et al. Helminth infections: the great neglected tropical diseases. J. Clin. Invest. 118, 1311-1321 (2008).

2. Allen, J. E. \& Maizels, R. M. Diversity and dialogue in immunity to helminths. Nature Rev. Immunol. 11, 375-388 (2011).

3. Herbert, D. R. et al. Intestinal epithelial cell secretion of RELM-beta protects against gastrointestinal worm infection. J. Exp. Med. 206, 2947-2957 (2009).

4. McKenzie, G. J., Bancroft, A., Grencis, R. K. \& McKenzie, A. N. A distinct role for interleukin-13 in Th2-cell-mediated immune responses. Curr. Biol. 8, 339-342 (1998).

5. Gerbe, F. et al. Distinct ATOH1 and Neurog3 requirements define tuft cells as a new secretory cell type in the intestinal epithelium. J. Cell Biol. 192, 767-780 (2011)

6. Bezençon, C. et al. Murine intestinal cells expressing Trpm5 are mostly brush cells and express markers of neuronal and inflammatory cells. J. Comp. Neurol. 509, 514-525 (2008).

7. Gerbe, F., Brulin, B., Makrini, L., Legraverend, C. \& Jay, P. DCAMKL-1 expression identifies tuft cells rather than stem cells in the adult mouse intestinal epithelium. Gastroenterology 137, 2179-2180 (2009). 
8. Bjerknes, M. et al. Origin of the brush cell lineage in the mouse intestinal epithelium. Dev. Biol. 362, 194-218 (2012).

9. Buczacki, S. J. et al. Intestinal label-retaining cells are secretory precursors expressing Lgr5. Nature 495, 65-69 (2013).

10. van Es, J. H. et al. Dll1 ${ }^{+}$secretory progenitor cells revert to stem cells upon crypt damage. Nature Cell Biol. 14, 1099-1104 (2012).

11. Gierl, M. S., Karoulias, N., Wende, H., Strehle, M. \& Birchmeier, C. The zinc-finger factor Insm1 (IA-1) is essential for the development of pancreatic $\beta$ cells and intestinal endocrine cells. Genes Dev. 20, 2465-2478 (2006).

12. Reynolds, L. A., Filbey, K. J. \& Maizels, R. M. Immunity to the model intestinal helminth parasite Heligmosomoides polygyrus. Semin. Immunopathol. 34, 829-846 (2012).

13. Ishikawa, N., Horii, Y. \& Nawa, Y. Immune-mediated alteration of the terminal sugars of goblet cell mucins in the small intestine of Nippostrongylus brasiliensis-infected rats. Immunology 78, 303-307 (1993).

14. Watanabe, N., Katakura, K., Kobayashi, A., Okumura, K. \& Ovary, Z. Protective immunity and eosinophilia in IgE-deficient SJA/9 mice infected with Nippostrongylus brasiliensis and Trichinella spiralis. Proc. Natl Acad. Sci. USA 85, 4460-4462 (1988).

15. Matsumoto, I., Ohmoto, M., Narukawa, M., Yoshihara, Y. \& Abe, K. Skn-1a (Pou2f3) specifies taste receptor cell lineage. Nature Neurosci. 14, 685-687 (2011).

16. Ohmoto, M. et al. Pou2f3/Skn-1a is necessary for the generation or differentiation of solitary chemosensory cells in the anterior nasal cavity. Biosci. Biotechnol. Biochem. 77, 2154-2156 (2013).

17. Yamaguchi, T. et al. Skn-1a/Pou $2 \mathrm{f3}$ is required for the generation of Trpm5-expressing microvillous cells in the mouse main olfactory epithelium. BMC Neurosci. 15, 13 (2014).

18. Moro, K. et al. Innate production of $T_{H} 2$ cytokines by adipose tissue-associated C-Kit ${ }^{+}$Sca- ${ }^{+}$lymphoid cells. Nature 463, 540-544 (2010).

19. Neill, D. R. et al. Nuocytes represent a new innate effector leukocyte that mediates type-2 immunity. Nature 464, 1367-1370 (2010).

20. Bastide, P. et al. Sox9 regulates cell proliferation and is required for Paneth cell differentiation in the intestinal epithelium. J. Cell Biol. 178, 635-648 (2007).

21. Blache, P. et al. SOX9 is an intestine crypt transcription factor, is regulated by the Wnt pathway, and represses the CDX2 and MUC2 genes. J. Cell Biol. 166, 37-47 (2004).

22. Mellitzer, G. et al. Loss of enteroendocrine cells in mice alters lipid absorption and glucose homeostasis and impairs postnatal survival. J. Clin. Invest. 120, 1708-1721 (2010)

23. Camberis, M., Le Gros, G. \& Urban, J. Jr. Animal model of Nippostrongylus brasiliensis and Heligmosomoides polygyrus. Curr. Protoc. Immunol. Ch. 19, Unit 19.12 (2003).

24. Artis, D. et al. RELM $\beta / F I Z Z 2$ is a goblet cell-specific immune-effector molecule in the gastrointestinal tract. Proc. Natl Acad. Sci. USA 101, 13596-13600 (2004).
25. Lawrence, R. A., Gray, C. A., Osborne, J. \& Maizels, R. M. Nippostrongylus brasiliensis: cytokine responses and nematode expulsion in normal and IL-4-deficient mice. Exp. Parasitol. 84, 65-73 (1996).

26. Urban, J. F. Jr et al. IL-13, IL-4R $\alpha$, and Stat6 are required for the expulsion of the gastrointestinal nematode parasite Nippostrongylus brasiliensis. Immunity 8, 255-264 (1998)

27. Sato, T. et al. Single Lgr5 stem cells build crypt-villus structures in vitro without a mesenchymal niche. Nature 459, 262-265 (2009).

28. Zhao, A. et al. Critical role of IL-25 in nematode infection-induced alterations in intestinal function. J. Immunol. 185, 6921-6929 (2010).

Supplementary Information is available in the online version of the paper.

Acknowledgements This work was supported by Association pour la Recherche contre le Cancer (ARC to N.T. and SL220110603456 to P.J.), Agence Nationale de la Recherche (ANR-09-BLAN-0368-01 to P.J., ANR-PolarAttack to V.D. and ANR-14-CE14-0025-01 to P.J. and N.T.), Institut National du Cancer (INCa 2014-174 to P.J.), a CNRS-NIH International Laboratory grant from the CNRS (LIA-BAGEL) to N.T. and the Welcome Trust (Ref. 106122 to R.M.M.). Part of the work was supported by institutional funds of Monell Chemical Senses Center to I.M.; E.S. and M.B. are supported by Ligue Nationale contre le Cancer, M.P. by the LabEx EpiGenMed and N.T. by Inserm. We are grateful to S. Gailhac and $\mathrm{C}$. Mongellaz for their expertise and assistance in immune cell analyses, S. Cording, J. Di Santo and G. Eberl for their generosity and their expertise on ILCs, G. Petrazzo and R. Guédon for technical input, S. Fre and M. Huygues for technical advice, C Legraverend for her expertise and to $M$. van de Wetering for reagents. We thank F. Gallardo, the PCEA and the IRD A2 facilities for maintenance of mouse colonies, the Montpellier RIO Imaging (MRI) facility, the Monell Histology and Cellular Localization Core (supported by funding from NIH Core Grant P3ODC011735 (to R. F. Margolskee, Monell Chemical Senses Center) for some of the histological analyses and Daniel Fisher for critical reading of the manuscript.

Author Contributions F.G. performed the majority of the experiments. E.S., D.J.S., B.B. and P.C. contributed to mouse studies, M.O. and I.M. to characterization of the Pou2f3-deficient mouse line, E.S., L.G. and M.B. to organoid experiments, V.D., M.P. and V.S.Z. to immune studies and Y.H. to parasite life cycle experiments. P.J. and F.G. conceived the study. P.J., F.G. and R.M.M. designed experiments with contributions from V.D., V.S.Z. and N.T.; P.J. wrote the manuscript with inputs from F.G. and N.T.

Author Information Reprints and permissions information is available at www.nature.com/reprints. The authors declare no competing financial interests. Readers are welcome to comment on the online version of the paper. Correspondence and requests for materials should be addressed to P.J. (philippe.jay@igf.cnrs.fr). 


\section{METHODS}

Animal strains. The Pou2f3-deficient mice (Pou2f3tm1Abek) have been previously described ${ }^{15}$. Il4r $\alpha$-deficient mice ${ }^{29}$ were provided by M. Kopf (Basel Institute for Immunology, Switzerland). C57BL/6 and BALB/c mice were obtained from Charles River Laboratories. All the mice were maintained in an SPF animal facility and were naive before the experiments. All animal experiments were approved by the Institutional Animal Care and Use Committee of Monell Chemical Senses Center or by the French Agriculture and Forestry Ministry. Unless specified, all mice were on a C57BL/ 6 genetic background. Mice were analysed at 10 weeks of age, regardless of the sex. For comparisons of wild-type and KO mice, littermates were analysed. Three mice per condition were analysed in all experiments. No statistical methods were used to predetermine sample size, no criteria of exclusion were defined and the experiments were not randomized nor blinded to the investigator. Immunophenotyping and flow cytometry analyses. Cells, isolated from peripheral lymph nodes, mesenteric lymph nodes, spleen and lamina propria were stained with Sytox blue or Live/dead fixable viability dye (Life Technologies and eBioscience respectively) together with the appropriate conjugated anti-CD3, CD45.2,

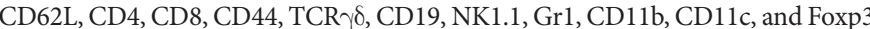
antibodies (eBioscience or Becton Dickinson, San Diego, CA). For ILC2 staining, cells were stained with a lineage cocktail and $\mathrm{CD} 45.2^{+}$(clone 104) and lineage-negative $\mathrm{CD} 45^{+}$cells were assessed for expression of CD127 (clone SB14), KLRG1 (clone 2F1), Sca-1 (clone D7), CD25 (clone 7D4), and intracellular expression of Gata-3 (clone L50-823). Th2 cells were identified on the basis of Gata-3-expressing cells within the $\mathrm{CD} 3^{+} \mathrm{CD} 4^{+}$subset.

IL-6, IL-12, TNF $\beta$, IL-10, MCP-1 and IFN- $\gamma$ production was assessed in the culture supernatant of LPS/IL-4-activated splenocytes using a Cytometric Bead Array (CBA) Kit (BD Biosciences). To assess intracellular cytokine production, freshly isolated and anti-CD3/CD28 stimulated LN cells were activated with PMA (SigmaAldrich; $100 \mathrm{ng} \mathrm{ml}^{-1}$ )/ionomycin (Sigma-Aldrich; $1 \mu \mathrm{g} \mathrm{ml}^{-1}$ ) in the presence of brefeldin A (Sigma-Aldrich; $10 \mu \mathrm{g} \mathrm{ml}^{-1}$ ) for $4 \mathrm{~h}$ at $37^{\circ} \mathrm{C}$, fixed, permeabilized and then stained with specific antibodies against IL- 2 and IFN- $\gamma$

Foxp3 staining was performed following fixation/permeabilization (eBioscience). Stained cells were assessed by flow cytometry (LSR Fortessa, Becton Dickinson, San Jose, CA) and data were analysed by FACSDiva (v.8.0, BD Biosciences) and FCAP Array Software (CBA analysis).

Ex vivo stimulations. $\mathrm{LN}$ cell activation was performed using plate-bound anti-CD3 (clone 2C11; $1 \mu \mathrm{g} \mathrm{ml}^{-1}$ ) and anti-CD28 (clone PV-1 $1 \mu \mathrm{g} \mathrm{ml}^{-1}$ ) mAbs in RPMI 1640 media (Life Technologies) supplemented with 10\% FCS, $2 \mathrm{mM}$ glutamine and $1 \%$ penicillin/streptomycin. Exogenous IL-2 $\left(100 \mathrm{U} \mathrm{ml}^{-1}\right)$ was added every other day starting at day 2 post-activation. Cell proliferation was monitored by labelling with CFSE (Life Technologies; $2.5 \mu \mathrm{M}$ ) for $3 \mathrm{~min}$ at room temperature. Splenocytes were activated with LPS $\left(20 \mu \mathrm{g} \mathrm{ml}^{-1}\right)$ and IL4 $\left(25 \mathrm{ng} \mathrm{ml}^{-1}\right)$. Supernatants were collected $40 \mathrm{~h}$ following activation.

Immunoglobulin detection. IgG detection in supernatants of LPS/IL-4-stimulated splenocytes was assayed by ELISA. Microtiter plates (Maxisorb, Nunc) were saturated overnight at $4{ }^{\circ} \mathrm{C}$ with $100 \mu \mathrm{l}$ of anti-IgG2a, anti-IgG2b, anti-IgA antibodies or anti-IgG $\left(\mathrm{Fab}^{\prime} 2\right)$ resuspended in PBS $\left(5 \mu \mathrm{g} \mathrm{ml}^{-1}\right)$. Plates were washed 3 times with $0.1 \%$ Tween-containing PBS (PBST). Samples ( $1 / 2$ dilution) were diluted in a final volume of $100 \mu \mathrm{l}$ per well of PBST-1\%BSA and incubated for $2 \mathrm{~h}$ at RT. Following washes, peroxidase-conjugated anti-mouse anti-IgG2a, anti-IgG2b, anti-IgA (Serotech) or anti-IgG gamma-chain (SIGMA) antibodies were added in PBST- $1 \%$ BSA (1:1,000 dilution; $100 \mu \mathrm{l}$ per well) and incubated for a $1 \mathrm{~h}$ at $37^{\circ} \mathrm{C}$. Immunoglobulin levels were then revealed with $o$-phenylenediamine (Sigma; $4 \mu \mathrm{g} \mathrm{ml}^{-1}$ ) in $0.1 \mathrm{M} \mathrm{Na}$ citrate and $003 \%$ hydrogen peroxide. Absorbance was measured at $450 \mathrm{~nm}$ using an automated plate reader (InfiniteM200Pro, TECAN) after $5 \mathrm{~min}$ at room temperature.

Tuft cell sorting. Freshly isolated small intestines of BC57BL/6 mice were flushed with PBS and incised along their length. The tissue was then incubated in $30 \mathrm{mM}$ EDTA (Sigma) in HBSS pH 7.4 (Life Technologies) on ice, and transferred in DMEM (Life Technologies) supplemented with 10\% FBS (Sigma). Vigorous shaking yielded the epithelial fraction that was then incubated with $100 \mu \mathrm{l}$ of Dispase (BD Biosciences) in $10 \mathrm{ml}$ of HBSS, supplemented with $100 \mu \mathrm{l}$ of DNase I at 2,000 Kunitz (Sigma). Single cell preparation obtained by filtration on a $30 \mu \mathrm{m}$ mesh was incubated with a phycoerythrin rat anti-mouse Siglec-F antibody for $30 \mathrm{~min}$ at $4{ }^{\circ} \mathrm{C}$ (BD pharmigen 552126), washed with HBSS and resuspended in appropriate volume of HBSS $\mathrm{pH} 7.4$ supplemented with 5\% FBS before staining with 7-aminoactinomycin D (Life Technologies) to exclude dead cells. Siglec- $\mathrm{F}^{+}$live cells were sorted using a FACSAria (Becton Dickinson), directly in RLT lysis buffer (Qiagen) for subsequent RNA extraction.

Parasite infections. Pou $2 \mathrm{f3}^{+/+}, \mathrm{Pou}_{2 \mathrm{f3}^{-/-}}, \mathrm{Il}_{4 \mathrm{ra}^{+/+}}$and $\mathrm{Il4ra}{ }^{-/-}, \mathrm{C} 57 \mathrm{BL} / 6$ and $\mathrm{BALB} / \mathrm{c}$ wild-type mice were used for $\mathrm{Nb}$ infection experiments. Mice were infected with $250 \mathrm{~L} 3$ infective $N b$ larvae by sub-cutaneous injection ${ }^{23}$ or with 200
H. polygyrus L3 larvae by gavage. Infection parameters were monitored by enumeration of live adult worms in the small intestinal tissue by two different investigators blinded to the study groups.

Reagents. Recombinant murine IL-4 (214-14), recombinant murine IL-13 (210-13) were purchased from PeproTech, and recombinant murine IL-25 (1399) was from R\&D Systems. For animal treatment, mice were injected intraperitoneally daily with a mixture of both interleukins or with rIL-25 $(40 \mu \mathrm{g}$ per $\mathrm{kg}$ of body weight). For rescue experiments in Pou $23^{-/-}$mice, rIL-25 was injected from day 3 post infection.

Organoid culture. Organoid cultures were performed as previously described ${ }^{27}$ using intestinal crypts from Pou $2 \mathrm{f3}^{+/+}$and Pou $2 \mathrm{f3}^{-/-}$mice. Organoid lines were passaged up to 10 times before experiments to ensure pure epithelial cultures. When indicated, cultures were stimulated with recombinant murine IL-4 (400 $\mathrm{n} \mathrm{ml}^{-1}$ ), recombinant murine IL-13 $\left(400 \mathrm{ng} \mathrm{ml}^{-1}\right)$ or an equimolar mixture of the two cytokines. For histological studies, organoids were washed twice in cold PBS to dissolve the Matrigel, fixed overnight in neutral-buffered formalin at $4^{\circ} \mathrm{C}$ and included in Histogel (Thermo Scientific) before paraffin embedding $(n=3$ experiments from independent mice).

RNA extraction and PCR. Total RNA from intestinal organoids or snap-frozen intestinal tissues was isolated using TRIzol (Life Technologies) followed by precipitation with isopropanol. Further RNA purification was carried out on RNeasy columns (Qiagen, 74104) and DNase treatment. In the case of Siglec ${ }^{+}$sorted tuft cells, extraction and DNase treatment were performed using RNeasy Micro KIT following the manufacturer's instructions. Reverse transcription was performed with $500 \mathrm{ng}-2 \mu \mathrm{g}$ of purified RNA using Transcriptor First Strand cDNA synthesis KIT (Roche) according to the manufacturer's instructions. For qRT-PCR experiments, gene expression was quantified on the LightCycler 480 using LightCycler 480 SYBR Green I Master (Roche). The results from three independent organoid cultures were normalized to the expression level of Gapdh and Hprt and relative expression was obtained using the $\Delta \Delta C_{\mathrm{t}}$ method. Primer sets for each gene are listed in Extended Data Table 1. PCR analyses were performed on an Eppendorf Mastercycler, using the primer sets listed in the Extended Data Table 1.

In situ hybridization. Single colorimetric and double fluorescent in situ hybridization analyses were carried out as described previously ${ }^{30}$. Briefly, digoxigenin- and fluorescein-labelled antisense RNAs were synthesized and used as probes after fragmentation to about 150 bases under alkaline conditions. Small intestines were dissected from mice shortly after euthanasia and embedded in frozen O.C.T. compound (Sakura Finetech). Fresh-frozen sections were prepared using a cryostat (CM1900, Leica Microsystems), fixed with 4\% paraformaldehyde, hybridized with probe(s), and then washed under stringent conditions. Hybridized probes were immunohistochemically detected using alkaline phosphatase-conjugated anti-digoxigenin antibody (Roche Diagnostics) and biotin-conjugated antifluorescein antibody (Vector Laboratories). Signals were developed using 4-nitro blue tetrazolium chloride/5-bromo-4-chloro-3-indolyl-phosphate as chromogenic substrates for single colorimetric analyses or the Tyramid Signal Amplification method and HNPP Fluorescent Detection Set (Roche Diagnostics) for doublefluorescent analyses. Stained and fluorescent images were obtained on a Nikon eclipse 80i microscope (Nikon Instruments Inc.) equipped with a DXM1200C digital camera (Nikon) and a Leica SP2 confocal scanning microscope (Leica), respectively. RNA probes generated were as follows: nucleotides $72-2363$ of Pou2f3 (GenBank accession number NM_011139), nucleotides 1-2228 of Slc15al (GenBank accession number BC116248), nucleotides 1-3255 of Muc2 (GenBank accession number BC034197), nucleotides 1-1102 of Gcg (GenBank accession number BC012975), nucleotides 1-584 of Gip (GenBank accession number BC104314), nucleotides 27-400 of Defcr6 (GenBank accession number M33225), nucleotides 1-1628 of Olfm4 (GenBank accession number BC141127), nucleotides 1-2750 of Dclk1 (GenBank accession number BC050903), and nucleotides 1-2797 of Ptgs1 (GenBank accession number BC005573).

Fluorescent and bright-field immunohistochemistry on paraffin-embedded tissue. Tissue dissection, fixation, and immunohistochemistry on thin sections of paraffin-embedded tissue were performed essentially as described previously ${ }^{5}$. Primary antibodies used in this study were as follows: anti-Sox 9 (AB5535; Millipore), anti-Cox1 (sc-1754; Santa Cruz), anti-PCNA (sc-56; Santa Cruz), anti-Plc $\gamma 2$ (sc-5283, Santa Cruz), anti-Gfilb (Sc-8559; Santa Cruz), anti Pou2f3 (sc-330, Santa Cruz and HPA019652, Prestige Antibodies), anti-Dclk1 (ab31704; AbCam), anti-Ki67 (ab16667; AbCam), anti-Retnlß (ABIN465494 Antibodies online), anti-IL-25 (mAb 1258; R\&D Systems). Anti-Insm1 was a gift from C. Birchmeier (Max-Delbrück-Center for Molecular Medicine; Berlin; Germany). Slides were washed twice times with 0.1\% PBS-Tween (Sigma-Aldrich) before incubation with fluorescent secondary antibodies conjugated with either Alexa 488, cyanin-3, or cyanin-5 (Jackson ImmunoResearch Laboratories, Inc.) and Hoechst at $2 \mu \mathrm{g} \mathrm{ml}^{-1}$ (Sigma-Aldrich) in PBS-Triton X-100 0.1\% 
(Sigma-Aldrich). Stained slides were washed again in PBS before mounting with FluoroMount (Sigma-Aldrich). Methods used for bright-field immunohistochemistry were identical, except that Envision+ (Dako) was used as a secondary reagent. Signals were developed with DAB (Sigma-Aldrich) and a haematoxylin counterstain (DiaPath) was used. After dehydration, sections were mounted in Pertex (Histolab). All experiments were performed on formalin-fixed tissues and $10 \mathrm{mM}$ sodium citrate ( $\mathrm{pH}$ 6.4) treated slides, except for IL-25 staining where Carnoy's fixation and $10 \mathrm{mM}$ Tris-EDTA ( $\mathrm{pH}$ 9.0) treatment were used. Enterocytes-alkaline phosphatase activity was revealed with Fast-red substrate (Sigma-Aldrich). Al stainings were repeated in 3 mice per group in 3 independent experiments and scored by three different investigators blinded to the study groups.

Microscopy and imaging. Fluorescent pictures were acquired at room temperature on an AxioImager Z1 microscope (Carl Zeiss, Inc.) equipped with a camera (AxioCam MRm; Carl Zeiss, Inc.), EC Plan Neofluar (5×, NA 0.16; 10×, NA 0.3; $20 \times, 0.5$ NA; $100 \times$, NA 1.3) and Plan Apochromat (40×, NA 0.95; 63×, NA 1.4) objectives, the Apotome Slider system equipped with an $\mathrm{H} 1$ transmission grid (Carl Zeiss, Inc.), and AxioVision software (Carl Zeiss, Inc.). Bright-field immunohistochemistry pictures were taken at room temperature on an Eclipse 80i microscope (Nikon) with Plan Fluor $(10 \times$, NA $0.3 ; 20 \times$, NA $0.5 ; 40 \times$, NA 0.75 ; and $60 \times$, NA 0.5-1.25) lenses (Nikon) and a digital camera (Q-Imaging Retiga 2000R with a Q-Imaging RGB Slider). Pictures were captured with Q-Capture Pro software (Nikon). Post-treatment of pictures (level correction), annotations, and panel composition were performed using Photoshop software (Adobe).
Statistical analyses. The Prism software was used for descriptive statistical analyses. For infection monitoring, sample $(n)$ was defined as the number of eggs per gram of faeces per mouse. As normal distribution assumption was not met, a twotailed Mann-Whitney $U$-test was used to calculate the $P$ value. For histological data quantification, sample $(n)$ was defined as the number of cells per crypt-villus unit Unless otherwise stated, 50 crypt-villus axes were counted per histological sections from 3 mice of each genotype or condition. According to the central limit theorem $(n>30)$, data comparison was achieved with a two-tailed Student's $t$-test. Welch's correction was applied to $P$-value calculation when homoscedasticity assumption was not met (determined with $F$-test).

Results are shown as histograms representing means as centre values and standard deviation as error bars for each genotype or conditions, except when $n<5$, where individual data point are plotted.

No statistical methods were used to predetermine sample size and the experiments were not randomized. Unless otherwise stated, the investigators were not blinded to allocation during experiments and outcome assessment.

29. Barner, M., Mohrs, M., Brombacher, F. \& Kopf, M. Differences between IL-4R $\alpha$ deficient and IL-4-deficient mice reveal a role for IL-13 in the regulation of Th2 responses. Curr. Biol. 8, 669-672 (1998).

30. Ohmoto, M., Matsumoto, I., Yasuoka, A., Yoshihara, Y. \& Abe, K. Genetic tracing of the gustatory and trigeminal neural pathways originating from T1R3expressing taste receptor cells and solitary chemoreceptor cells. Mol. Cell. Neurosci. 38, 505-517 (2008). 


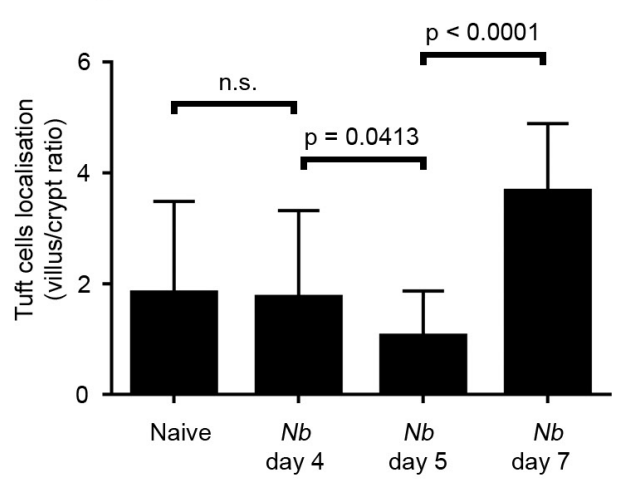

b

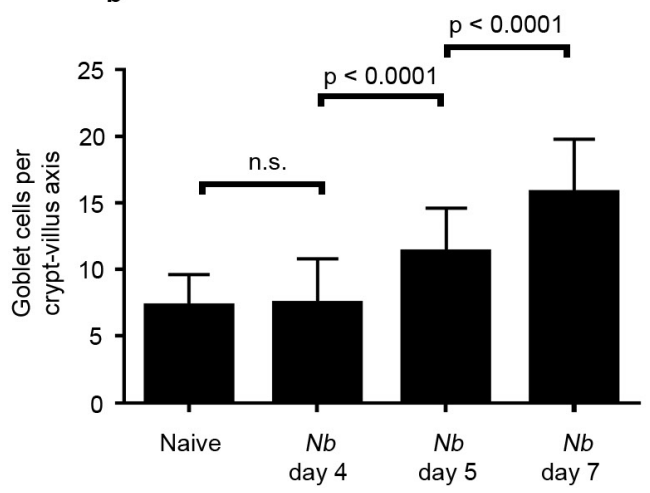

c

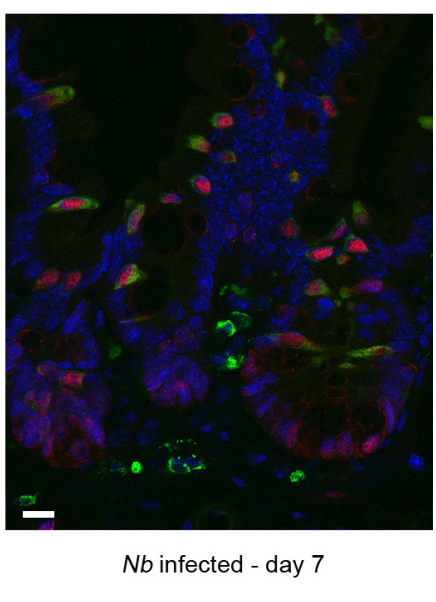

d

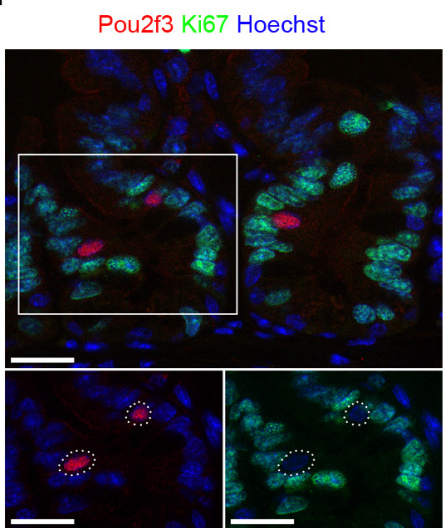

f
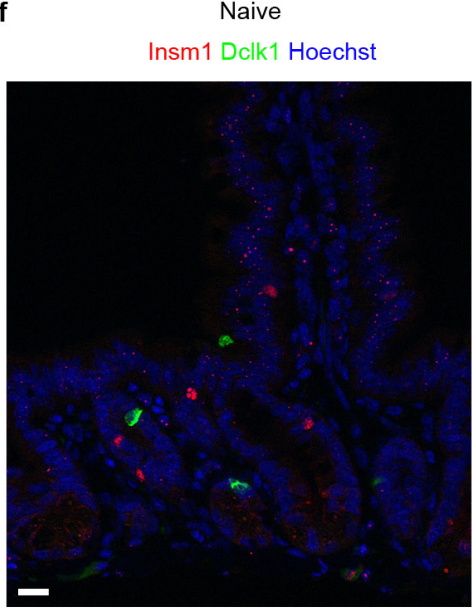

Extended Data Figure 1 | Epithelial differentiation parameters during $N \boldsymbol{b}$ infection. a, Graph showing the distribution of Dclk $1^{+}$tuft cells in naive and infected mice 4, 5 and 7 days post infection. Cells were counted in the crypt and villus compartments of $n=50$ crypt-villus units per mouse with 3 mice per condition. Means of villus/crypt ratio of tuft cell numbers are shown. $\mathbf{b}$, Quantification of the goblet cell hyperplasia in naive and infected mice 4,5 and 7 days post infection ( $n=50$ crypt-villus units per mouse; 3 mice per condition). c, Neo-differentiating tuft cells following $\mathrm{Nb}$ infection are indistinguishable from the tuft cells found in naive mouse intestinal epithelium as shown with Sox 9 and $\mathrm{Plc} \gamma 2$ stainings $\left(n=3\right.$ mice). d, Proliferation status of Pou $2 \mathrm{f}^{+}{ }^{+}$tuft cells in naive and
$\mathrm{Nb}$ infected - day 7

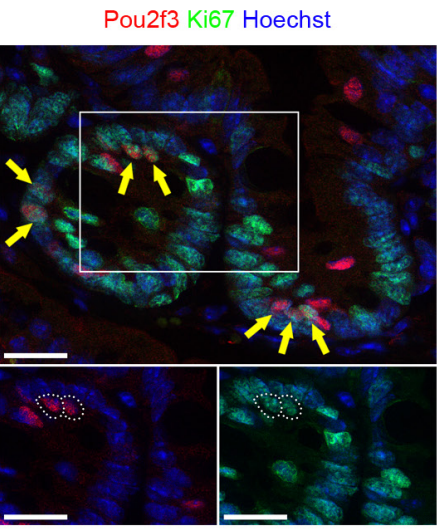

Nb infected - day 7

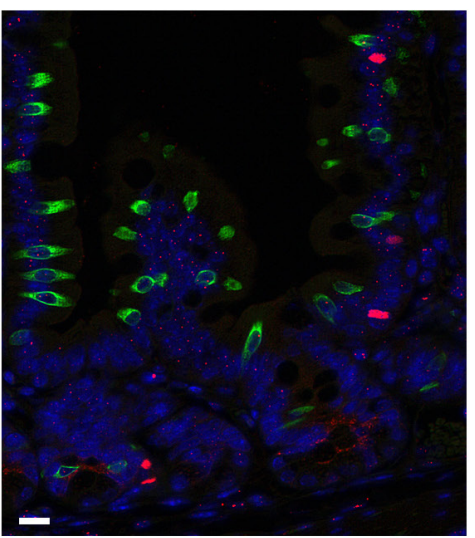

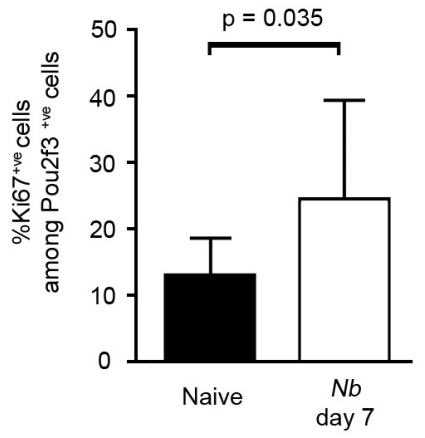

g

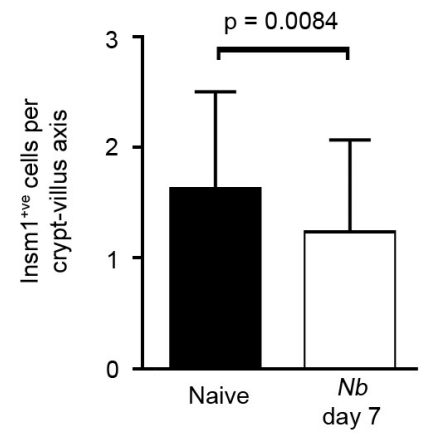

infected mice, shown with co-expression with the Ki67 proliferation marker. Arrows indicate $\mathrm{Ki}_{67^{+}}$cells located at various positions along the crypt axis. e, Increased proliferation of Pou $2 \mathrm{f}^{+}{ }^{+}$tuft cells during response to $\mathrm{Nb}$ infection ( $n=3$ naive and 3 infected mice). f, Dclk $1^{+}$tuft cells and Insm $1^{+}$enteroendocrine cells are distinct populations ( $n=3$ mice). g, Decrease of the Insm $1^{+}$enteroendocrine cell population during type 2 responses to $\mathrm{Nb}$ infection, concomitant to the expansion of the tuft cell lineage 7 days post infection ( $n=3$ naive and 3 infected mice). All the histograms show means \pm s.d. A two-tailed Student's $t$-test with Welch's correction was used, except for $\mathbf{g}$ where the 2 groups displayed comparable variances. All stainings were repeated 3 times. 


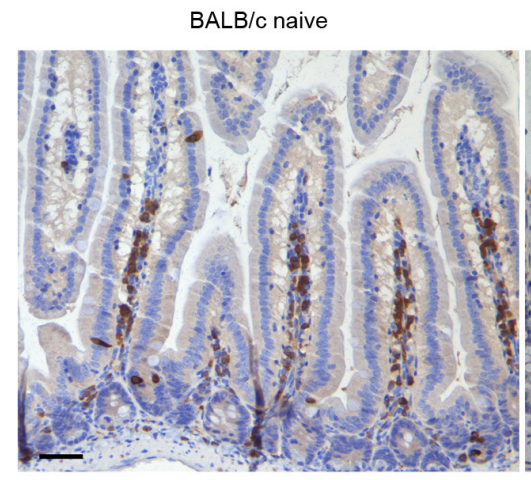

BALB/c Nb day 8

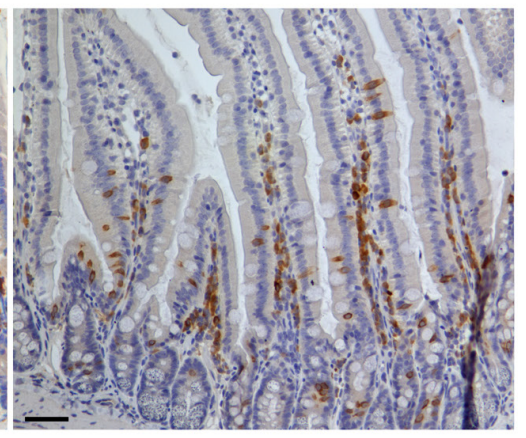

b

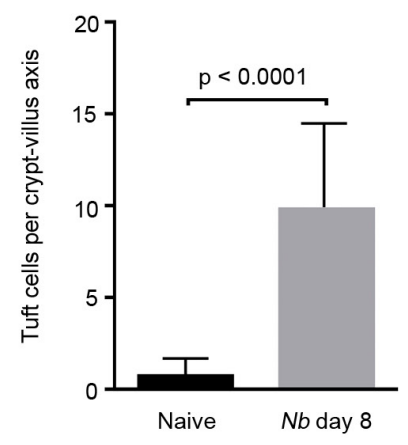

d

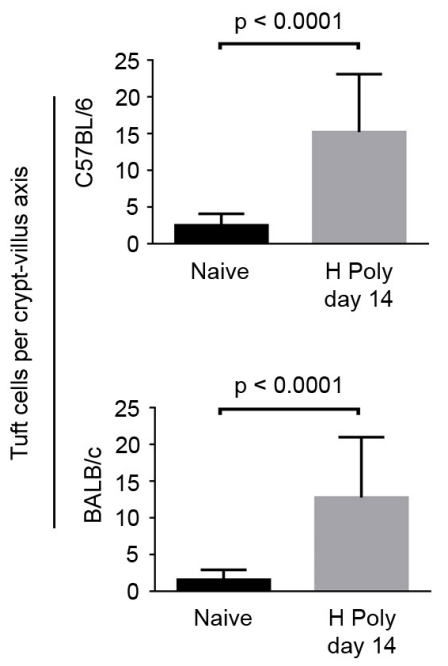

C

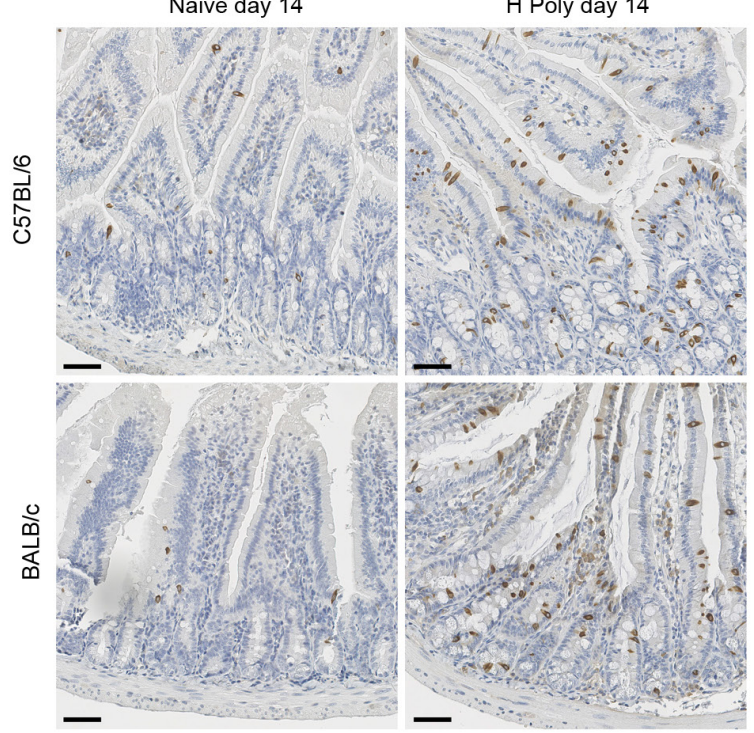

Nb infected day 6

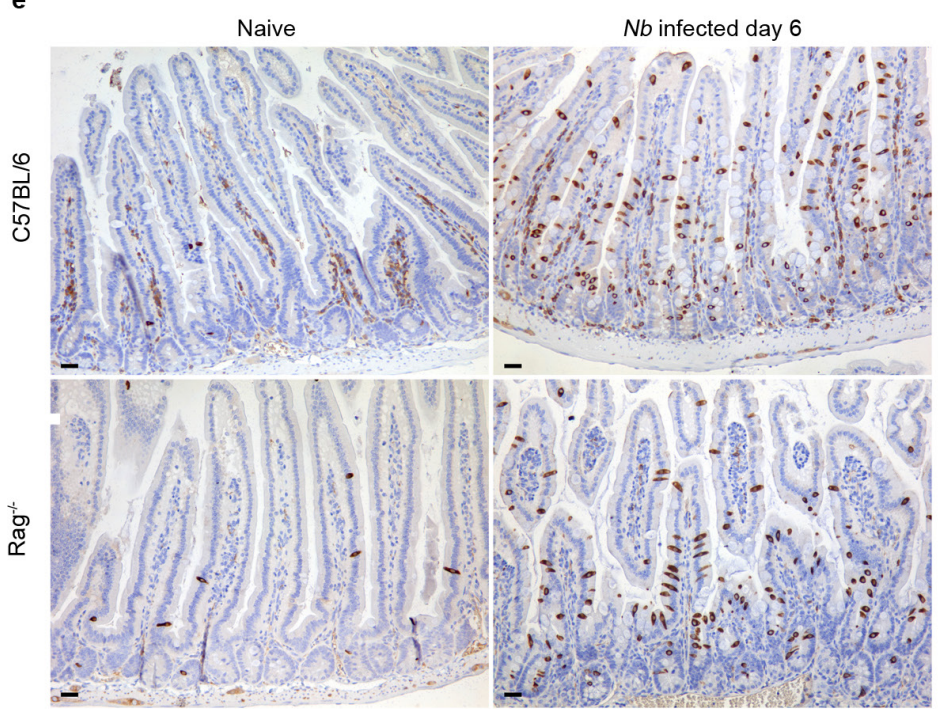

f

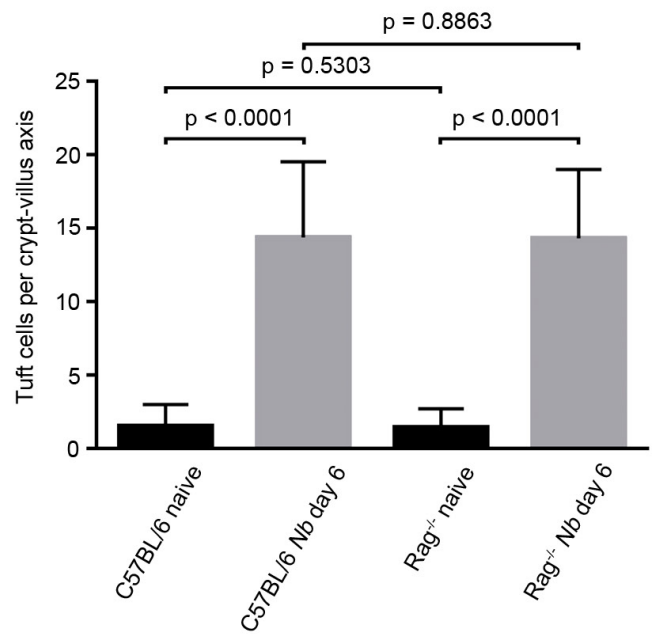

Extended Data Figure 2 Expansion of the tuft cell lineage is a common adaptation of the intestinal epithelium following infection with helminth parasites. Tuft cell lineage expansion was assessed by Dclk1 immunohistochemistry in 2 different genetic backgrounds following infection with two different helminths, at the indicated time points. a, b, Naive and $N b$-infected BALB/c mice. c, d, Naive and
H. polygyrus-infected C57BL/6 and BALB/c mice. e, f, Naive and $\mathrm{Nb}$-infected C57BL/ 6 and $\mathrm{Rag}^{-/-}$mice. b, d, f, $n=50$ crypt-villus units per mouse; 3 mice per condition. Data are shown as means \pm s.d. and $P$ values are indicated. A two-tailed Student's $t$-test with Welch's correction was used. Scale bars, $20 \mu \mathrm{m}$. All experiments displayed in this figure were repeated 3 times. 


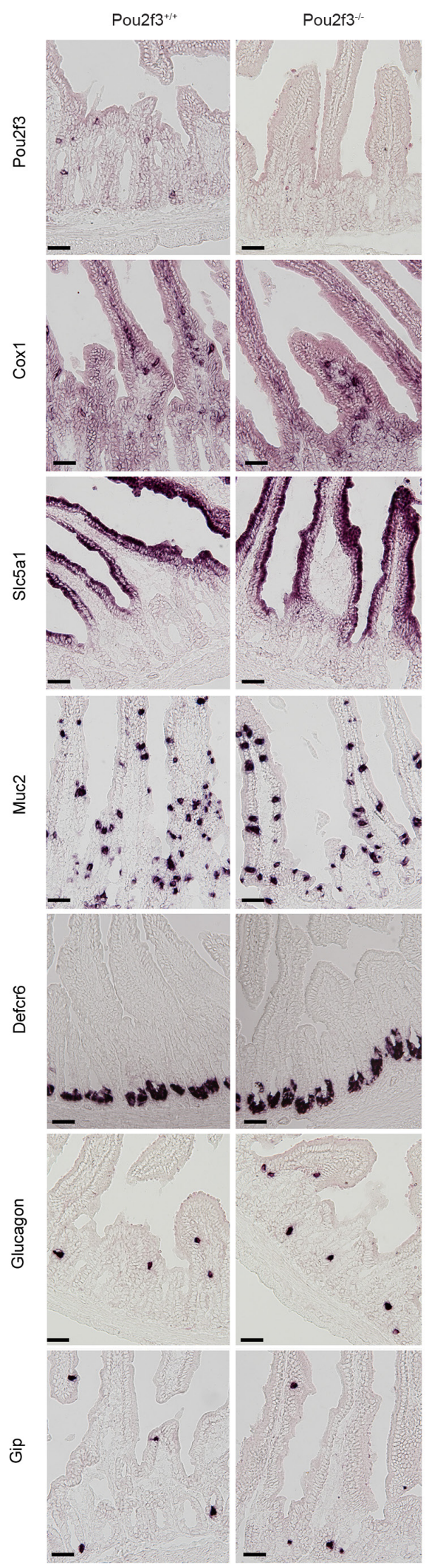

Extended Data Figure $3 \mid$ Pou2f3 deficiency results in the specific absence of tuft cells in the intestinal epithelium. Characterization of the intestinal epithelium in Pou2f3-deficient mice as compared with wild-type littermate controls ( $n=3$ mice of each genotype). Left, in situ hybridization showing absence of tuft cells (Pou2f3, Cox 1) in Pou $2 \mathrm{f3}^{-1}$ mice, whereas enterocytes (Slc5a1), goblet cells (Muc2), Paneth cells (Defcr6) and enteroendocrine cells (glucagon, Gip) are unaffected. Right, representative pictures of the in situ hybridization $(\mathrm{Olfm} 4)$ and

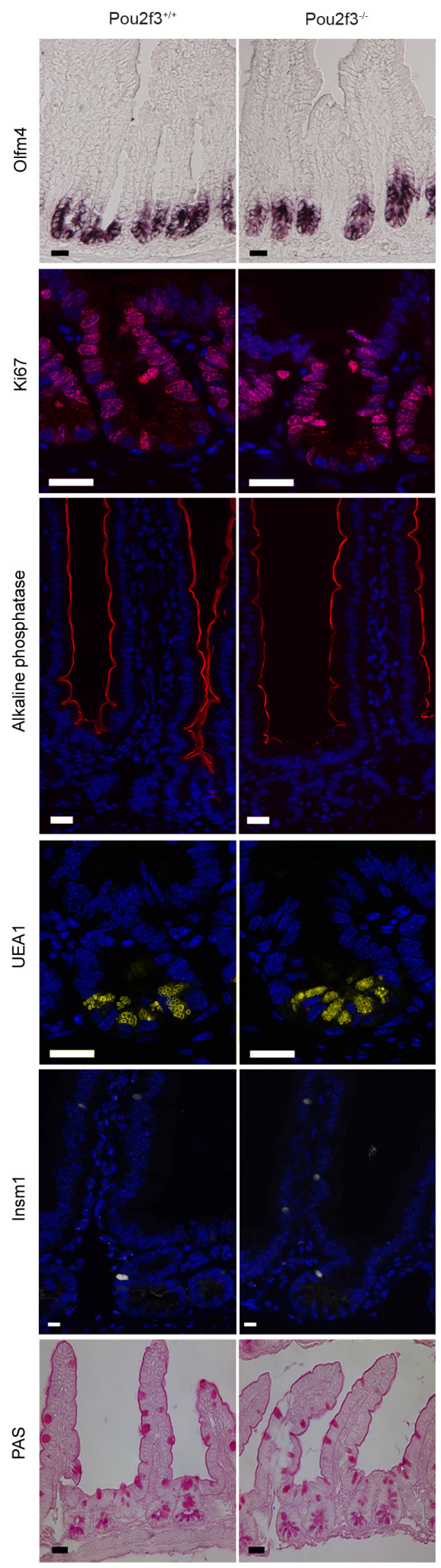

immunohistochemistry experiments underlying the quantitative analysis provided in Fig. 2c showing that the stem cells (Olfm4), proliferative compartment (Ki67), and differentiated cell types: enterocytes (alkaline phosphatase), Paneth (UEA1), enteroendocrine (Insm1) and goblet (PAS staining) cells populations are unaffected in the Pou $23^{-1-}$ mice. All panels show representative pictures of experiments replicated 3 times in 3 different mice. Scale bars, $20 \mu \mathrm{m}$. 
a

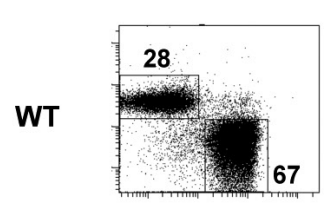

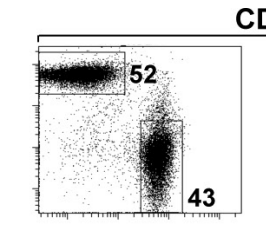

CD3 $^{+}$
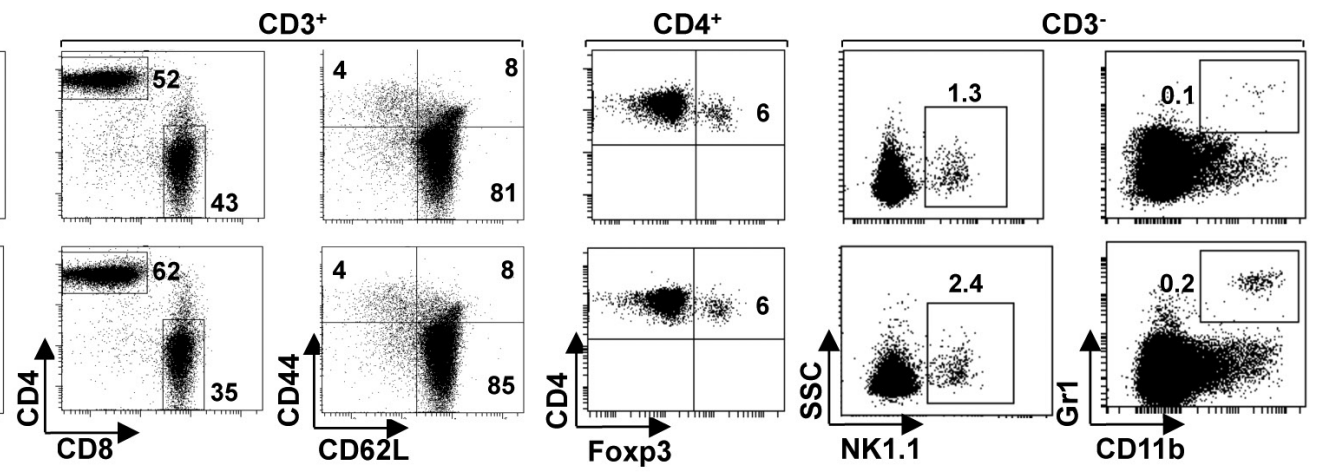

b
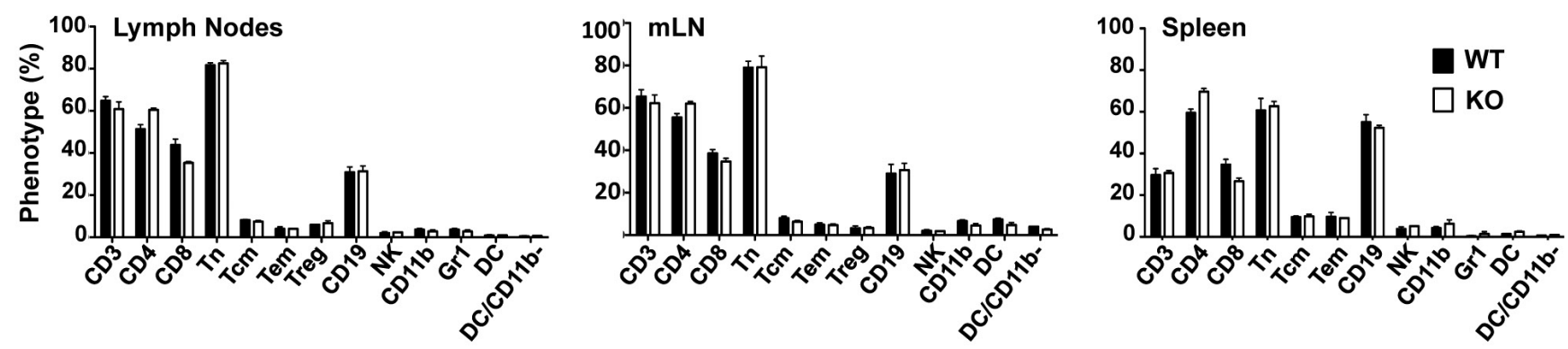

C

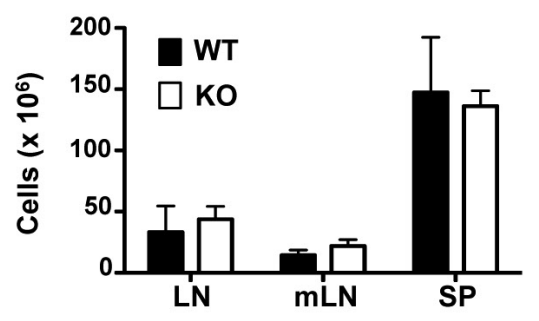

d
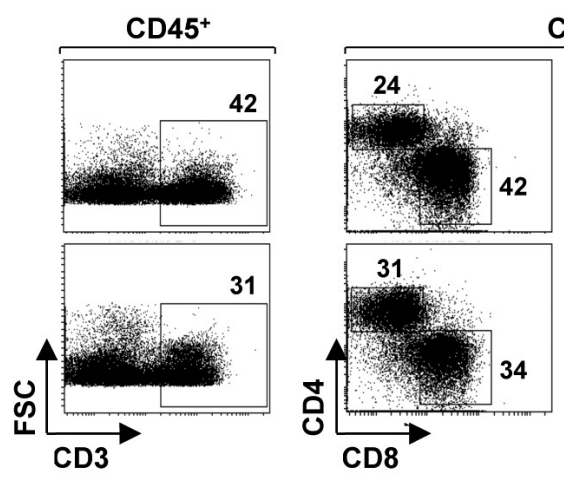

$\mathrm{CD}^{+}$

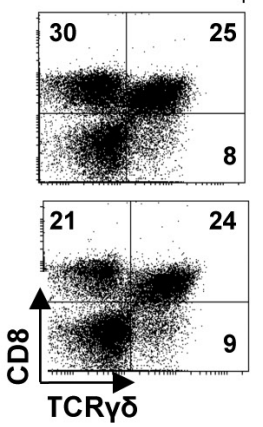

Extended Data Figure $4 \mid$ Immune cell homeostasis is not altered in Pou $23^{-/-}$tuft-cell-deficient mice. a, The repartition of immune cells in wild-type and Pou $2 \mathrm{f3}^{-/-}$mice was monitored by flow cytometry. The presence of $\mathrm{T}\left(\mathrm{CD}^{+}\right), \mathrm{B}\left(\mathrm{CD} 19^{+}\right), \mathrm{CD} 4^{+}, \mathrm{CD} 8^{+}$, naive $\left(\mathrm{Tn}\right.$; $\mathrm{CD}^{+}$ $\left.\mathrm{CD} 62 \mathrm{~L}^{+} \mathrm{CD} 44^{-}\right)$, central memory $\left(\mathrm{Tcm} ; \mathrm{CD}^{+}{ }^{\mathrm{CD}} 62 \mathrm{~L}^{+} \mathrm{CD} 44^{+}\right)$, effector memory (Tem; $\mathrm{CD}^{+} \mathrm{CD}_{2} \mathrm{~L}^{-} \mathrm{CD} 44^{+}$), regulatory $\left(\right.$Treg; $\mathrm{CD} 4{ }^{+} \mathrm{Foxp}^{+}$), natural killer $\left(\mathrm{NK} ; \mathrm{CD}^{-} \mathrm{NK} 1.1^{+}\right)$and myeloid $\left(\mathrm{CD} 11 \mathrm{~b}^{+} \mathrm{Gr} 1^{+}\right)$cells was assessed by staining with fluorochrome-tagged antibodies and representative dot plots are shown. The percentages of positively-stained cells are indicated. $\mathbf{b}$, Quantification of the different immune cells in lymph nodes (LN), mesenteric lymph nodes ( $\mathrm{mLN}$ ) and spleens (SP) of wild-type and Pou $f 3^{-/-}$mice are presented. Data are means \pm s.d.
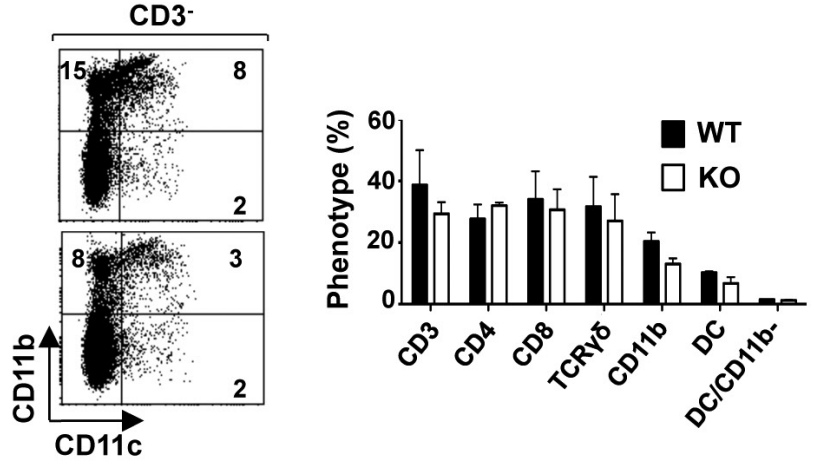

( $n=3$ mice per genotype). $\mathrm{c}$, Total cells in LN, $\mathrm{mLN}$ and SP of wild-type and Pou $2 \mathrm{f}^{-/-}$mice are presented as means \pm s.d. $(n=3$ mice per genotype). d, Immune lineage cells in the lamina propria of wild-type and Pou $23^{-/-}$mice were monitored by flow cytometry after tissue dissociation. The percentage of T cells was assessed within the CD $45^{+}$ haematopoietic gate, CD4, CD8 and gamma-delta T cells $\left(\mathrm{CD} 8^{+} \mathrm{TCR}-\gamma \delta^{+}\right)$ within the $\mathrm{CD}^{+}$gate and myeloid cells within the $\mathrm{CD}^{-}$gate, as indicated. Representative dot plots are presented (left). Quantification of immune cells within the lamina propria are shown as means \pm s.d. ( $n=3$ mice per group). No significant differences were detected for all cell types between wild-type and Pou $23^{-1-}$ mice $(P>0.05)$. A two-tailed Student's $t$-test was used. 
a
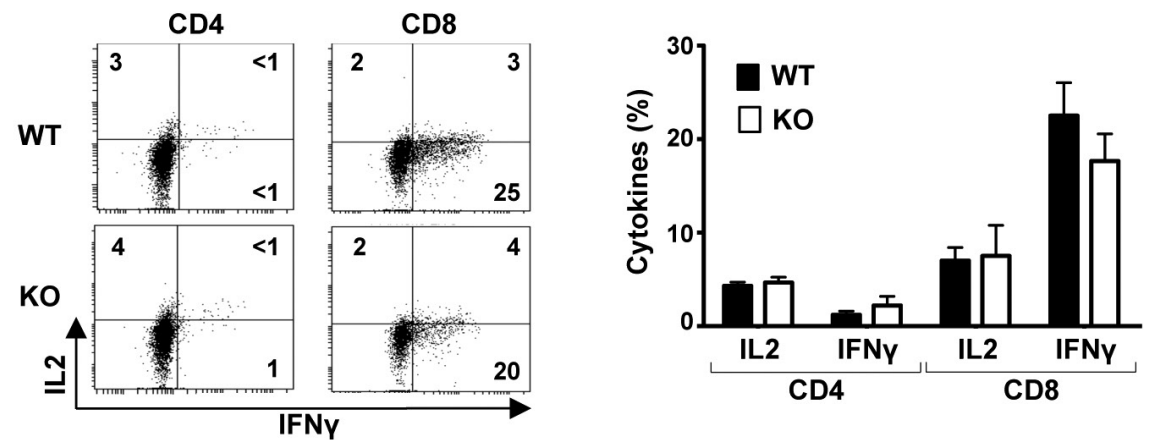

b

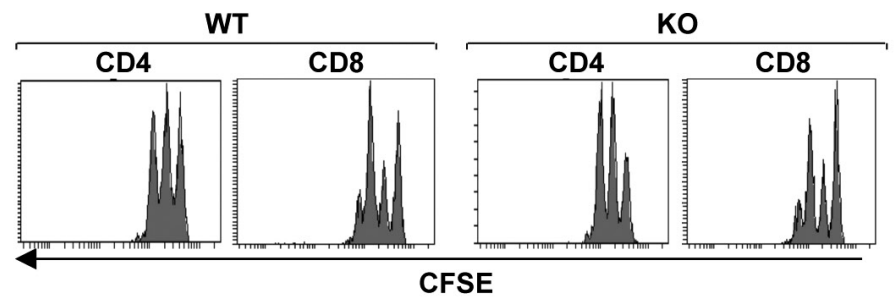

C
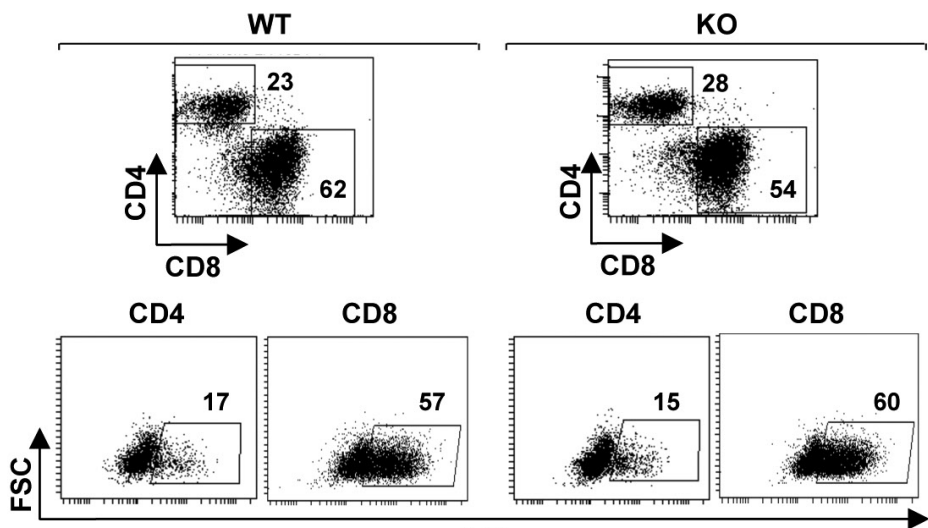

IFNY d

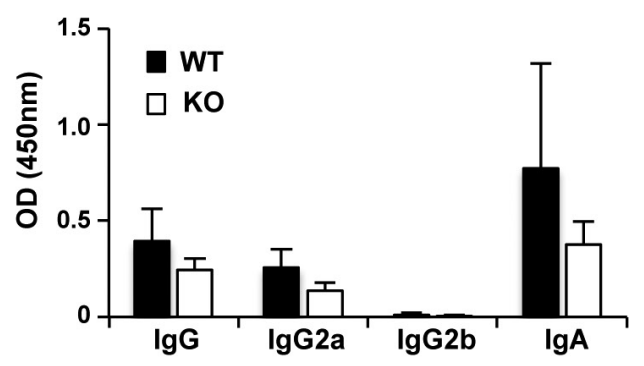

e

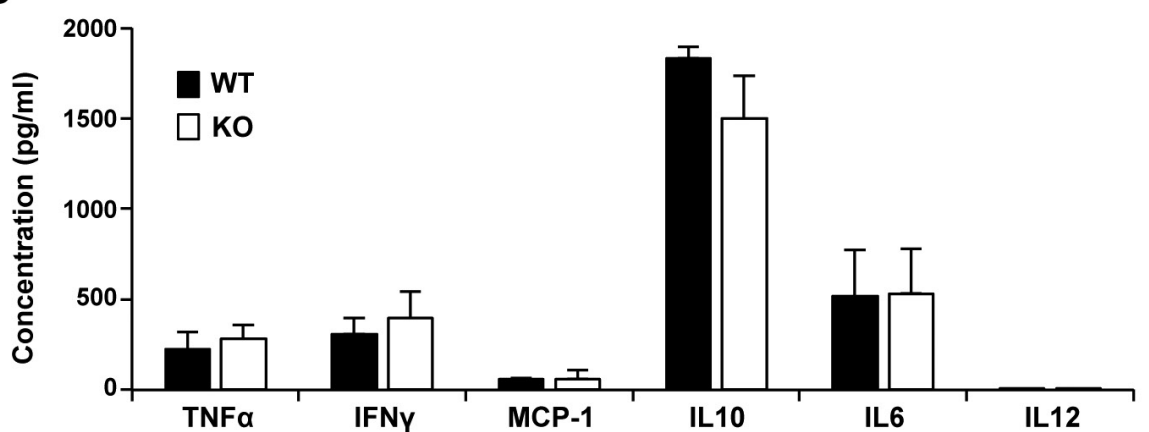

Extended Data Figure 5 Equivalent immune responsiveness of wild-type and Pou $\mathrm{f}^{-/-}$lymphocytes. a, The level of IL-2 and interferon gamma (IFN- $\gamma$ ) production by Pou $2 \mathrm{f}^{+/+}$and Pou $2 \mathrm{f3}^{-/-} \mathrm{CD} 4$ and CD8 lymph node $\mathrm{T}$ cells was monitored directly after ex vivo isolation and representative histograms are presented (left). Quantification of cytokine secreting CD4 and CD8 T cells are presented as means \pm s.d. $(n=3$ per group; $P>0.05)$. b. CFSE-loaded T cells were activated with immobilized anti-CD3/anti-CD28 antibodies for 2 days and proliferation was monitored as a function of fluorescence dilution. Representative histograms for CD4 and CD8 T cells are shown. c, IFN- $\gamma$ production in wild-type and Pou $2 \mathrm{f3}^{-/-}$lymphocytes was assessed at day 6 post CD3/CD28 stimulation and representative plots for CD4 and CD8 T cells are presented. d, Splenocytes from wild-type and Pou $23^{-1-}$ mice were activated with LPS+IL-4 for $40 \mathrm{~h}$ and levels of secreted IgG, IgG2a, $\mathrm{IgG} 2 \mathrm{~b}$ and IgA were monitored by ELISA. Means \pm s.d. are presented. e, Splenocytes were activated as above and levels of TNF- $\alpha$, IFN- $\gamma$, MCP-1, IL-10, IL-6, and IL-12 were monitored by cytometric bead array. Means \pm s.d. are presented. A two-tailed Student's $t$-test was used. 
a

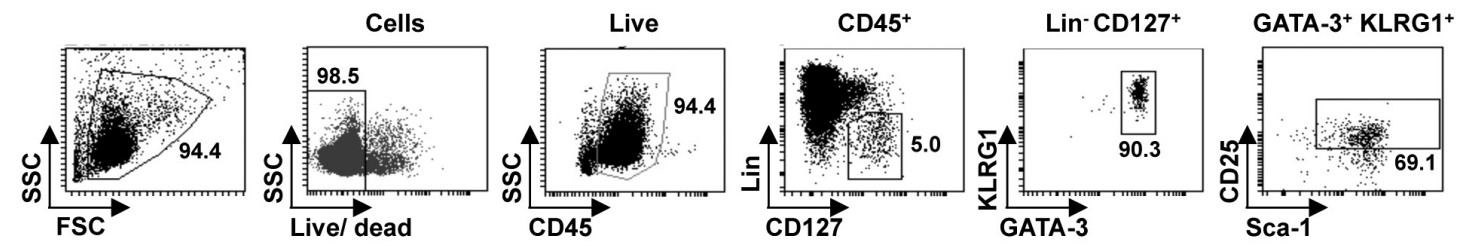

b

Mesenteric Lymph Nodes (mLN)

C

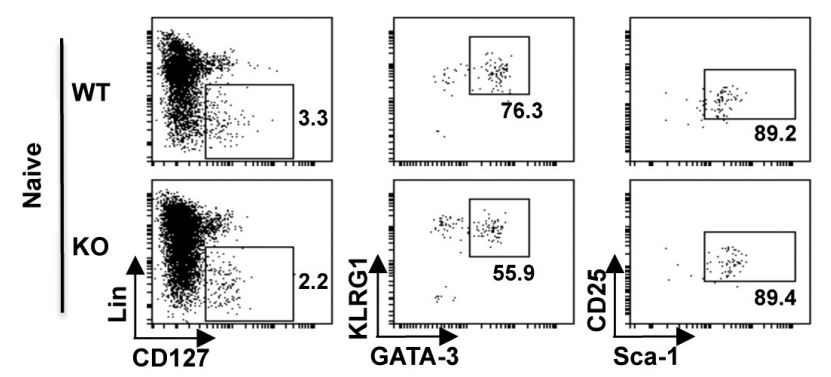

d
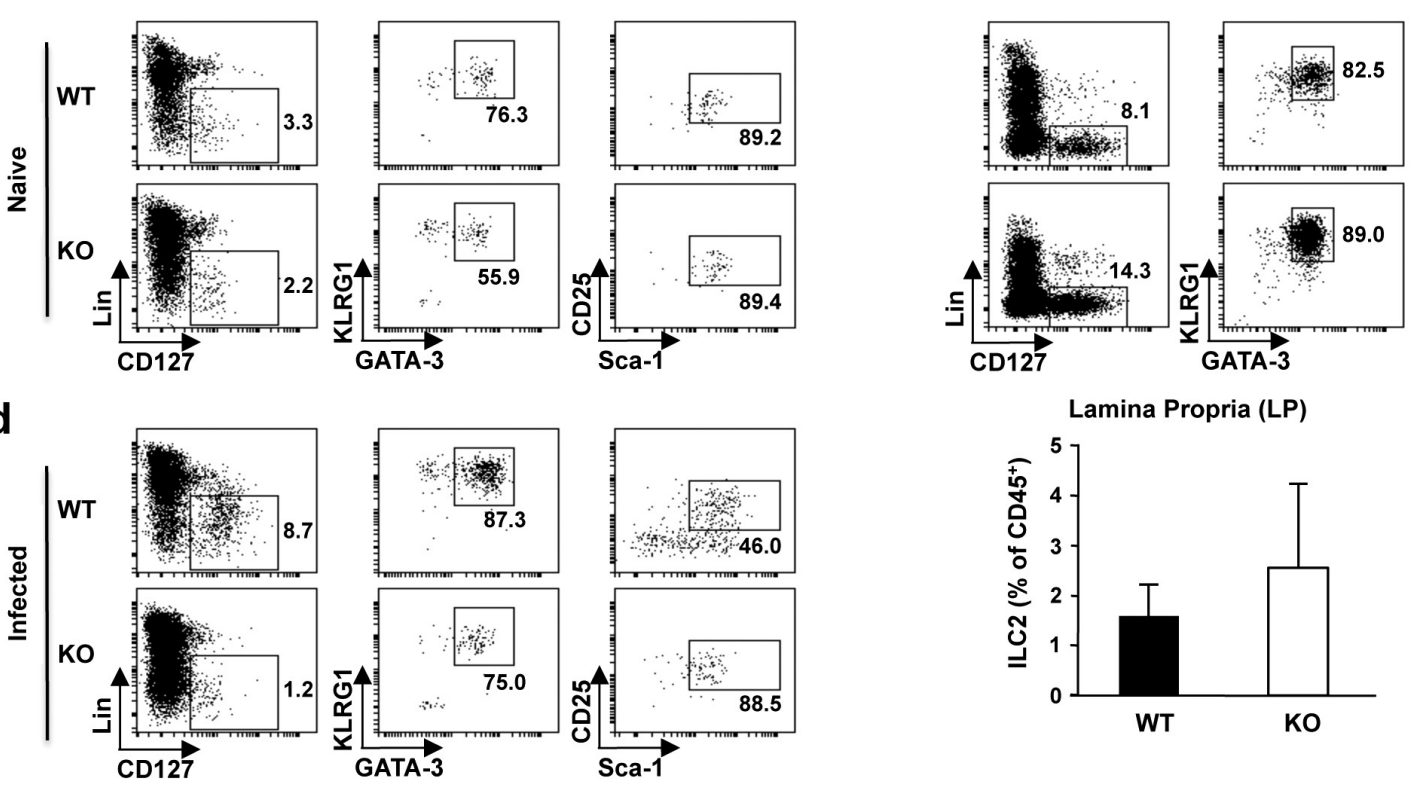

e
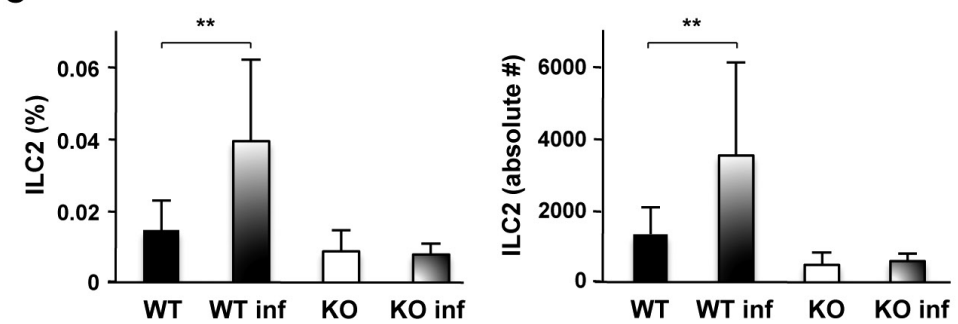

$\mathbf{f}$

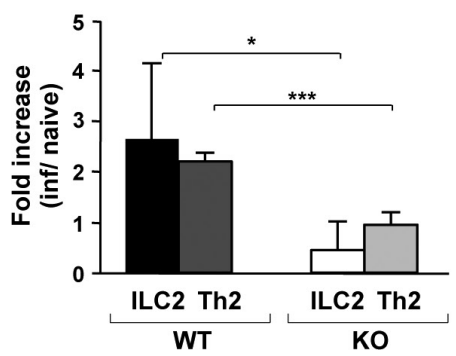

Extended Data Figure 6 | Defective induction of type 2 immunity in Pou $23^{-/-}$mice following helminth infection. a, Flow cytometry gating strategy for analysis of the innate ILC2 subset is shown. ILC2s were assessed within the $\mathrm{CD} 45^{+}$haematopoietic subset as lineage-CD $127^{+}$ cells expressing KLRG1, GATA-3, Sca-1 and CD25 cell surface markers. Numbers represent the percentages of boxed cells. The staining strategy was validated using $\mathrm{mLN}$ cells from ZAP- $70^{-1-}$ mice as this subset is present at relatively high levels in these immunodeficient mice. b. The presence of ILC2 cells in mLNs of naive Pou $23^{+/+}(\mathrm{WT})$ and Pou $2 \mathrm{f3}^{-/-}(\mathrm{KO})$ mice was assessed using the gating strategy shown above. Representative data from WT $(n=8)$ and $\mathrm{KO}(n=5)$ mice are presented. $c$, Representative plots of ILC2 cells in lamina propria of naive
WT $(n=7)$ and KO $(n=5)$ mice are shown (top). Quantifications of ILC2 are presented as means \pm s.d. d, WT and KO mice were infected with $N$. brasiliensis and the presence of ILC2 in mLNs was assessed 5 days post infection. Representative plots are shown ( $n=6$ mice per group). e, Quantification of ILC2 cells in mLN of naive versus infected WT and KO mice. The percentage of ILC2s within the live gate (left) and the absolute numbers of ILC2s (right) are presented. Data are means \pm s.d. ( $n=5$ for $\mathrm{WT}, n=8$ for KO, $n=6$ for both groups of infected mice). $* * P=0.01 . \mathbf{f}$, The fold-increase in ILC2 (lineage ${ }^{-} \mathrm{CD} 127^{+} \mathrm{KLRG}^{+}{ }^{+} \mathrm{GATA}-3^{+}$) and Th2 $\left(\mathrm{CD}^{+}{ }^{+} \mathrm{CD} 4^{+} \mathrm{Gata}-3^{+}\right)$cells in mLN was assessed as a function of infection ( $n=6$ per group). The mean fold-increase \pm s.d. in $\mathrm{WT}$ and $\mathrm{KO}$ mice is presented. $* P=0.02, * * * P=0.0005$. A two-tailed Student's $t$-test was used. 

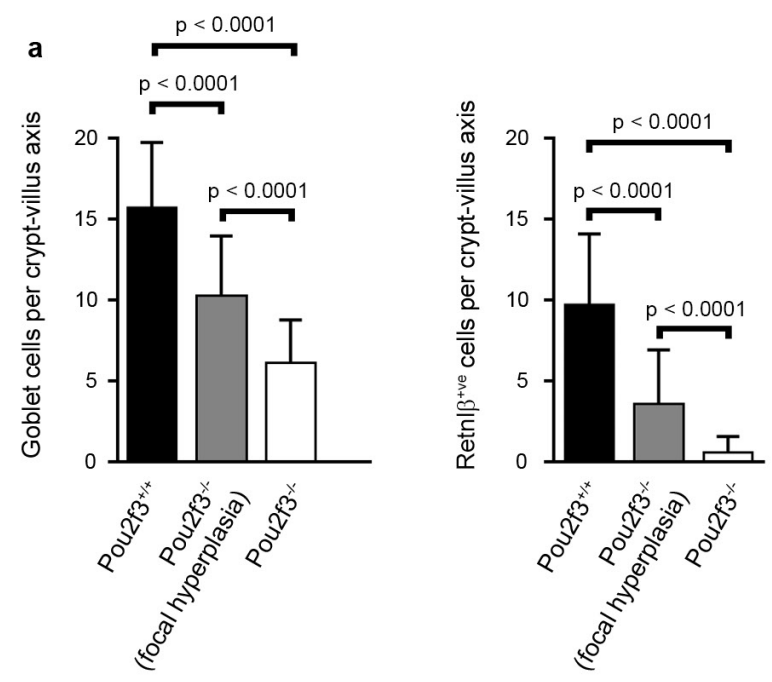

b

C

$$
\text { Pou } 23^{+/+}
$$

Pou2f3-1-
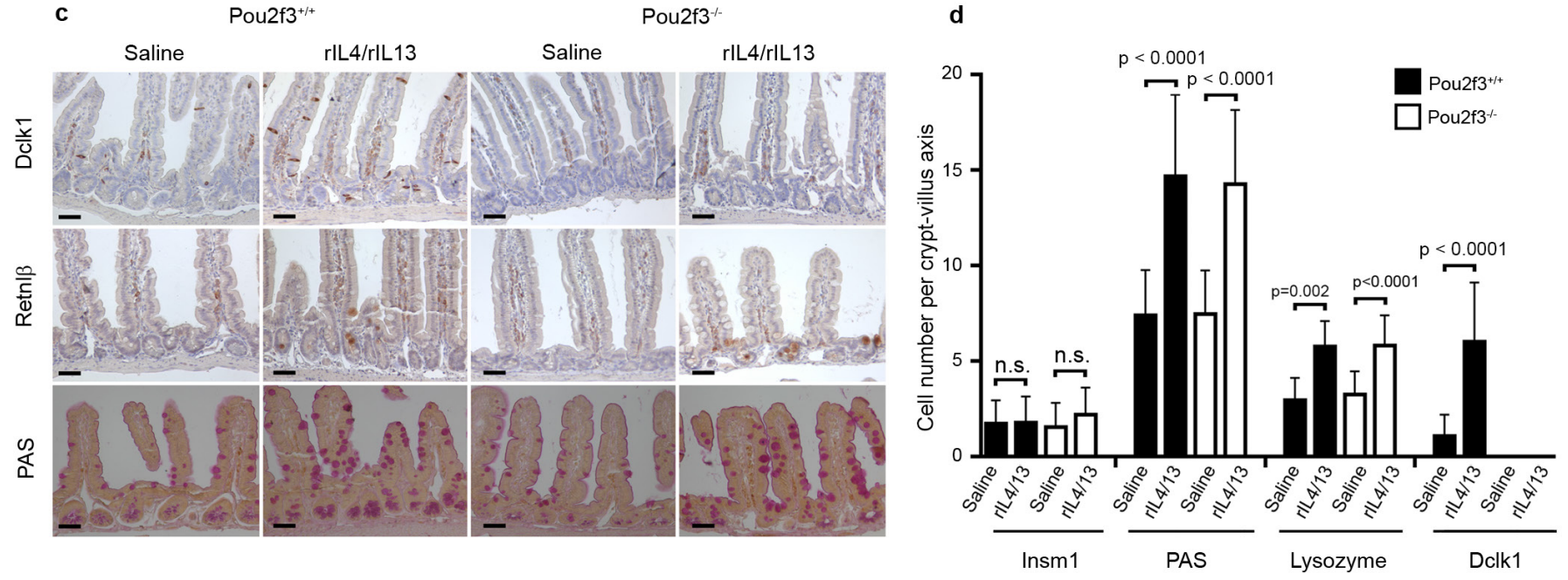

Extended Data Figure 7 | Signalling via IL-4R $\alpha$ is required and sufficient to induce goblet and tuft cell hyperplasia. a, Quantification of goblet (PAS and Retnl $\beta$ staining) cells in Pou $2 \mathrm{f3}^{+/+}$and Pou $2 \mathrm{f3}^{-/-}$mice infected with $\mathrm{Nb}$ (day 7 post infection). In Pou $2 \mathrm{f3}^{-/-}$mice, crypt-villus axes from both focally responding regions and the rest of the tissue were counted. b, Quantification of tuft cells (Dclk1 staining) and goblet cell hyperplasia (PAS) in $I l 4 r \alpha^{+/+}$and $I l 4 r \alpha^{-/-}$mice. c, Histological analysis showing tuft (Dclk1 staining) and goblet (PAS and Retnl $\beta$ staining) cells in Pou $23^{+1+}$ and Pou $23^{-1-}$ mice following treatment with a mixture
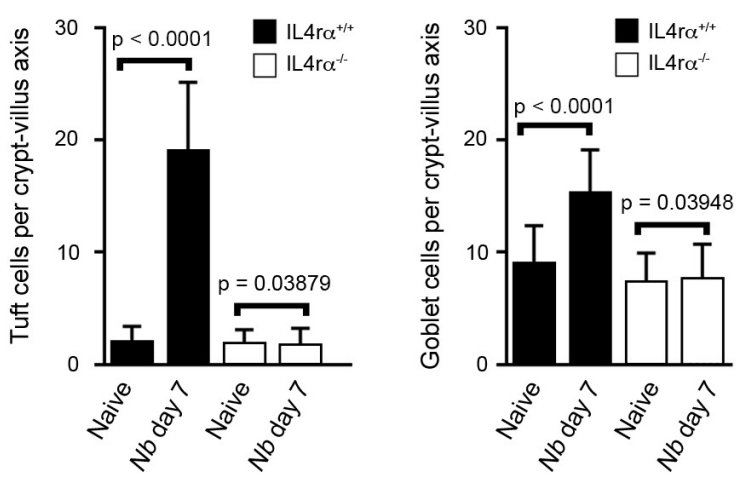

of rIL- 4 and rIL- 13 for 5 days. $n=3$ mice per condition. All panels show representative experiments replicated 3 times. Scale bars, $20 \mu \mathrm{m}$. d, Quantitative analysis of the changes in the different cell types of the intestinal epithelium of Pou $2 \mathrm{f3}^{+/+}$and Pou $23^{-/-}$mice following treatment with a mixture of rIL- 4 and rIL-13 during 5 days. For $\mathbf{a}, \mathbf{b}, \mathbf{d}, n=50$ crypt-villus axes counted in 3 mice per genotype or condition. Data are shown as means \pm s.d. and $P$ values are indicated. A two-tailed Student's $t$-test with Welch's correction was used. 


\section{RESEARCH LETTER}

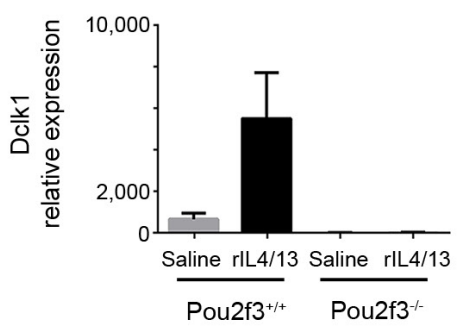

b

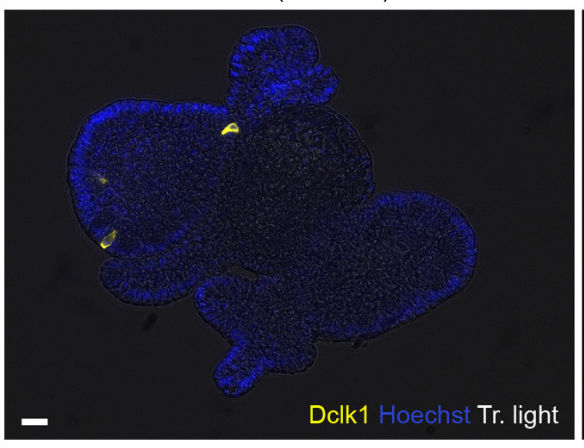

rlL4/IL13 (48 hours)

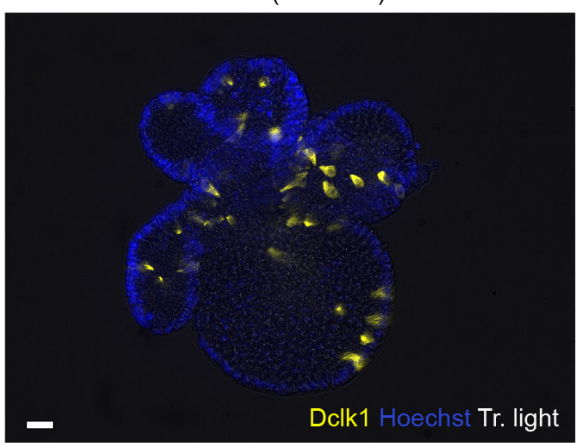

。
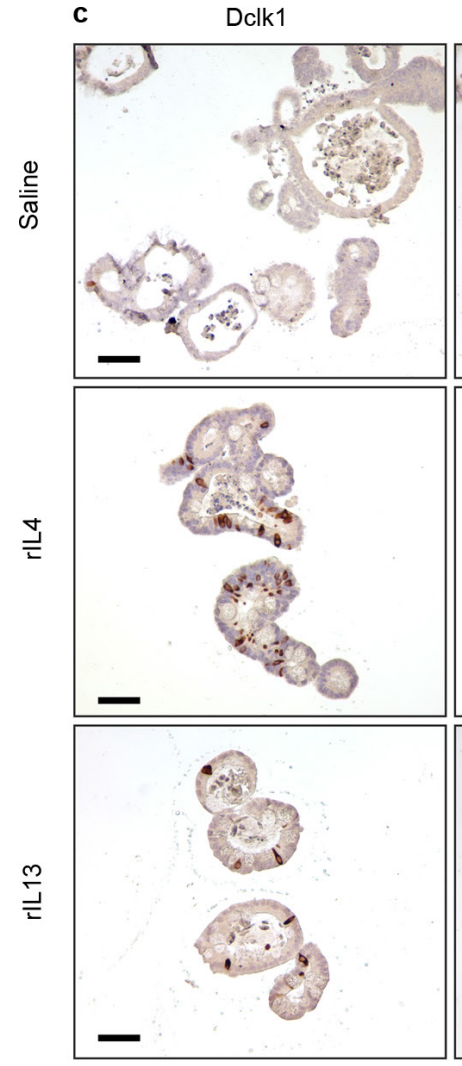

Extended Data Figure 8 | Signalling via IL-4R $\alpha$ is sufficient to induce goblet and tuft cell hyperplasia in mouse intestinal organoids. a, Quantification of Dclk1 expression analysis by qRT-PCR in Pou $23^{+/+}$ and Pou $2 \mathrm{f3}^{-1-}$ organoids following rIL-4/rIL-13 treatment for $48 \mathrm{~h}$ to assess the presence and amplification of tuft cells. Means \pm s.d., relative to Gapdh and Hprt, are presented. b, Expansion of the tuft cell lineage in wild-type organoids following rIL-4/rIL-13 administration ( $48 \mathrm{~h})$

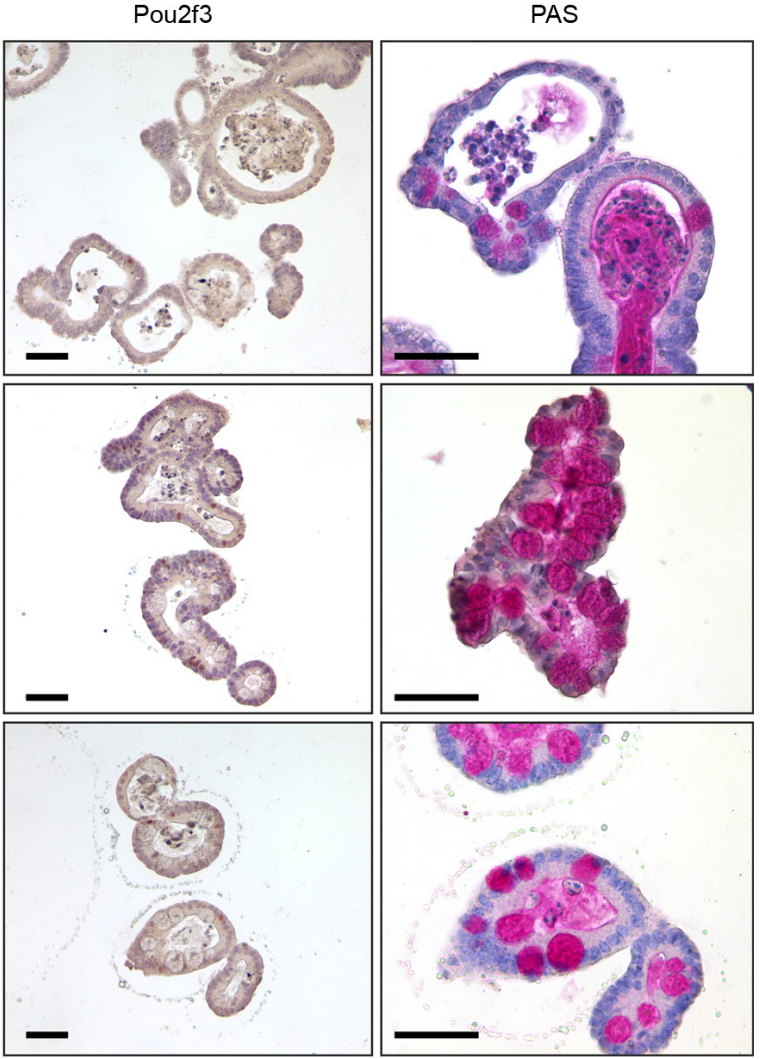

d

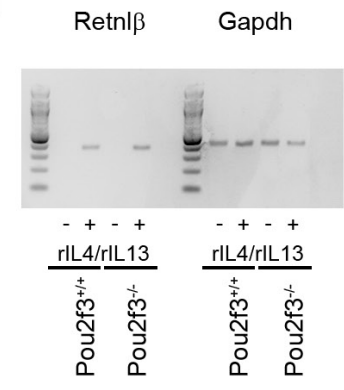

was monitored by Dclk1 staining. c, Expansion of the tuft cell lineage in wild-type organoids following IL-4 or IL-13 administration ( $48 \mathrm{~h}$ ) was monitored by Dclk1, Pou2f3 and PAS stainings. Scale bars, $20 \mu \mathrm{m}$.

d, Retnl $\beta$ expression in Pou $23^{+/+}$and Pou $23^{-/-}$organoids was monitored as a function of rIL- $4 / \mathrm{rIL}-13$ treatment $(48 \mathrm{~h})$ by RT-PCR and data relative to Gapdh are presented. All panels show representative experiments from 3 independent organoid cultures, replicated 3 times. 


\section{LETTER RESEARCH}

a

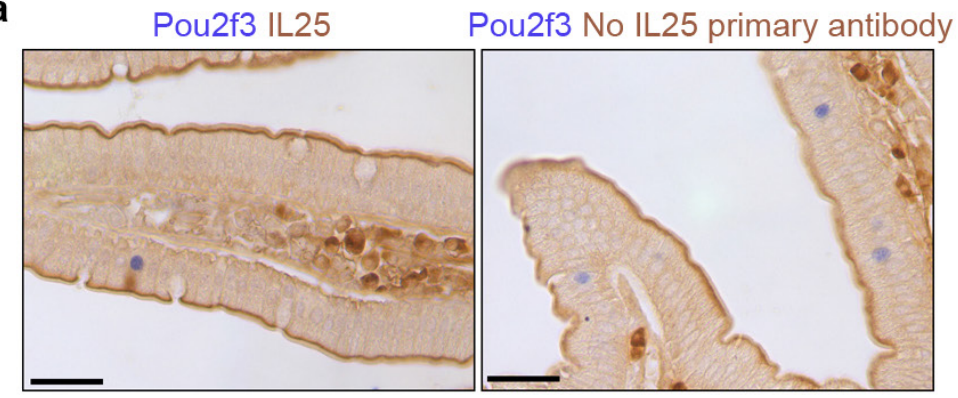

b

Dclk1 SiglecF Hoechst

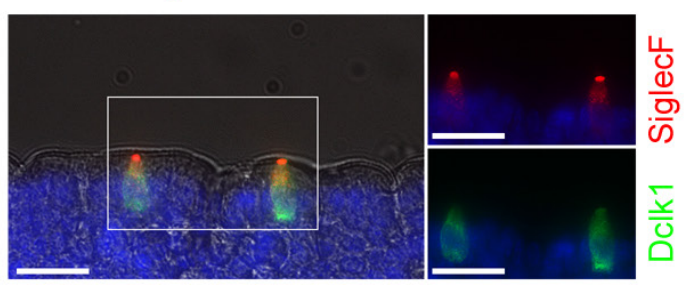

Extended Data Figure 9 | Validation of the Siglec-F and IL-25 stainings. a, Control experiment for specificity of the IL-25 immunohistochemistry, in presence (left) or absence (right) of IL-25 primary antibody. b. Immunohistochemistry showing specificity of Siglec-F as a marker for intestinal epithelial tuft cells. All panels show representative experiments from 3 independent mice, replicated 3 times. 


\section{RESEARCH LETTER}

Extended Data Table 1 | List of the oligonucleotide primer sequences

Table1: Primer sets used for qPCR analyses ( $\left.5^{\prime} 3^{\prime}\right)$ :

\begin{tabular}{|l|l|l|}
\hline Dclk1 & CAGCCTGGACGAGCTGGTGG & TGACCAGTTGGGGTTCACAT \\
\hline Gapdh & GGAGCGAGACCCCACTAACA & ACATACTCAGCACCGGCCTC \\
\hline Hprt & GCAGTACAGCCCCAAAATGG & GGTCCTTTTCACCAGCAAGCT \\
\hline
\end{tabular}

Table 2: Primer sets used for PCR analyses $\left(5^{\prime} 3^{\prime}\right)$ :

\begin{tabular}{|l|l|l|}
\hline IL13 & AGCTCCCTGGTTCTCTCACT & CTCATTAGAAGGGGCCGTGG \\
\hline IL25 & TCTTGGCAATGATCGTGGGA & TGTGGTAAAGTGGGACGGAG \\
\hline Retnlb & CCAGAAGACCATTTCCTGAGCT & CCCACGATCCACAGCCATAG \\
\hline Gapdh & CAAGAAGGTGGTGAAGCAGG & TCAAGAGAGTAGGGAGGGCT \\
\hline Pou2f3 & AGAGAATCAACTGCCCCGTG & GGAAGGCACGACTCTCTTCC \\
\hline
\end{tabular}

